\title{
STEIN SHRINKAGE AND SECOND-ORDER EFFICIENCY FOR SEMIPARAMETRIC ESTIMATION OF THE SHIFT
}

\author{
ARNAK S. DALALYAN
}

\begin{abstract}
The problem of estimating the shift (or, equivalently, the center of symmetry) of an unknown symmetric and periodic function $f$ observed in Gaussian white noise is considered. Using the blockwise Stein method, a penalized profile likelihood with a data-driven penalization is introduced so that the estimator of the center of symmetry is defined as the maximizer of the penalized profile likelihood. This estimator has the advantage of being independent of the functional class to which the signal $f$ si assumed to belong and, furthermore, is shown to be semiparametrically adaptive and efficient.

Moreover, the second-order term of the risk expansion of the proposed estimator is proved to behave at least as well as the second-order term of the risk of the best possible estimator using monotone smoothing filter. Under mild assumptions, this estimator is shown to be second-order minimax sharp adaptive over the whole scale of Sobolev balls with smoothness $\beta>1$. Thus, these results extend those of [10, where second-order asymptotic minimaxity is proved for an estimator depending on the functional class containing $f$ and $\beta \geq 2$ is required.
\end{abstract}

\section{InTRODUCTION}

1.1. Motivation. The increasing interest to the semiparametric models in recent years is mainly explained by the compromise they offer between the relative simplicity of parametric inference and the flexibility of nonparametric modeling. In many semiparametric models, though the ignorance of an infinite dimensional nuisance parameter, the finite-dimensional parameter of interest can be estimated as well as if the nuisance parameter were known. In most situations, there are many estimators having this feature. Moreover, most of them depend on the functional class to which the unknown nuisance parameter is assumed to belong.

The aim of the present paper is to propose a second-order efficient and entirely datadependent estimator in the problem of shift estimation when the observed shifted signal is corrupted by the Gaussian white noise. This problem has been studied in [10, and the goal of the present paper is to complete the inference developed there. In particular, the estimator proposed in [10] and proved to be second-order minimax when the signal belongs to a Sobolev ball, depends on the parameters of the Sobolev ball in question. In the present work, we propose an estimator of the

Date: August 23, 2018.

2000 Mathematics Subject Classification. 62G05, 62G20.

Key words and phrases. semiparametric estimation, second-order efficiency, penalized profile likelihood, exact minimax asymptotics, Stein shrinkage, adaptive smoothing. 
shift parameter that is independent of the parameters of the Sobolev ball and is second-order minimax simultaneously for a large scale of Sobolev balls.

To further motivate the study of the second-order efficiency in semiparametric inference, let us briefly recall a popular general method of construction of efficient estimators in a semiparametric model: the profile likelihood maximization. The asymptotic properties of this method are studied in 32 and, in a more general fashion, in 24. Profile likelihood techniques are effectively applied in a number of contexts such as laser vibrometry signals [22, varying coefficient partially linear models $[13$, and so forth.

If the parameter of interest is partitioned as $(\vartheta ; f)$, with $\vartheta$ being a low-dimensional parameter of interest and $f$ a higher dimensional nuisance parameter, and $l_{n}(\vartheta ; f)$ is the log-likelihood of the model, then the profile likelihood for $\vartheta$ is defined as $p l_{n}(\vartheta)=\sup _{f \in \mathcal{F}} l_{n}(\vartheta ; f)$ and the Profile Likelihood Estimator (PLE) is $\vartheta_{P L E}=$ $\arg \max _{\vartheta} p l_{n}(\vartheta)$. Thus, the nuisance parameter $f$ is eliminated by taking the sup over all possible values of $f$ in some a priori chosen class $\mathcal{F}$.

A natural question arises: what is the best way of choosing the class $\mathrm{F}$ and what is the impact of this choice on the accuracy of the PLE? The theory fails to answer this question as long as only the first-order term of the risk is considered. It seems that the most appealing way to study the dependence on $\mathcal{F}$ of the accuracy of the PLE is to consider the second-order term of the quadratic risk. This approach is developed in [16, 17] for partial linear models, in [10] for a nonlinear model with a shift parameter, and in 7 for a model with scaling parameter. In these papers, the second-order asymptotic minimaxity of the proposed estimators is proved and optimal constants are obtained.

Another important point is that the study of the second-order asymptotics of the risk allows one to refine the choice of the tuning parameters, if there are, of the estimating procedure. This argument is used in [19, 21, 23. in order to propose a rate-optimal choice of a tuning parameter.

The results of the above mentioned papers grant an increasing importance to the second-order terms in that they show that, in a semiparametric estimation problem, the second-order term is not dramatically smaller than the first-order term, especially when the nuisance parameter is not very smooth (or not very sparse). Thus, the investigation of second-order efficient estimators is not only a challenging theoretical problem, but is also of practical interest.

1.2. Methodology. The main goal of the present paper is to define an estimator of $\vartheta$ which adapts automatically to the smoothness of $f$ and is simultaneously second-order efficient over a large variety of Sobolev balls. For linear models, such a procedure has firstly been proposed by Golubev and Härdle in [17. They use the well known idea of unbiased risk minimization in order to determine the data driven filter. However, their procedure is provably second-order efficient only when the (data driven) filter and the contrast function are based on independent subsamples. This sample-splitting technique is frequently used in theory (see e.g. 2, [26, p. 19]), but it is rather unattractive from the practical point of view.

In this paper, we focus on the shift estimation of a periodic symmetric signal and use the celebrated Stein shrinkage (see 20, 29]) for defining the data-driven version of 
the penalized profile likelihood. While there is a huge number of papers concerning the Stein's shrinkage in nonparametric estimation (4, 5, 6, 8, 9, 11, 28, see also 34 for a comprehensive discussion), this is to our knowledge the first paper where Stein's shrinkage is applied in the context of semiparametric estimation.

The construction we use is closer to the one of 9 in that a penalized version of the Stein estimator with weakly geometrically increasing blocks is considered. It seems that, unlike in nonparametric inference, in semiparametric inference it is crucial to use the penalized shrinkage in order to get the second-order efficiency. An important advantage of using the Stein Shrinkage is that, unlike the procedure proposed in [17, our procedure is not based on the sample-splitting technique and, nevertheless, enjoys the property of second-order asymptotic minimaxity.

Note also that our results hold when the function $f$ has arbitrary Sobolev smoothness $\beta>1$. However, for $\beta<2$, the penalized profile likelihood (PPL) is not necessarily concave over the whole parameter space, therefore we use a two-step procedure. We first construct an initial $\sqrt{n}$-consistant estimator $\bar{\vartheta}$ of $\vartheta$, and then maximize the PPL over a shrinking neighborhood of $\bar{\vartheta}$, where we prove that the PPL is concave with probability close to one.

1.3. Structure of the paper. Section 2 describes the model and introduces the Penalized Maximum Likelihood Estimator (PMLE) based on a filtering sequence $h$. In Section 3, the local concavity of the PPL is proved and the PMLE based on a data dependent choice of $h$ is proposed. Oracle inequalities for adaptive PMLE and its second-order efficiency over Sobolev balls are stated and proved in Section 4. Finally, Section 5 contains the definition of a preliminary estimator and the technical details of the proofs.

\section{A Simple Semiparametric MOdel}

Consider the "signal in Gaussian white noise model", that is the observations $\left(x^{\varepsilon}(t), t \in[-1 / 2,1 / 2]\right)$ with

$$
d x^{\varepsilon}(t)=f_{\vartheta}(t) d t+\varepsilon d W(t), \quad t \in[-1 / 2,1 / 2],
$$

are available, where $W(t)$ is a Brownian motion. Assume that the signal has the form $f_{\vartheta}(t)=f(t-\vartheta)$, where $f: \mathbb{R} \rightarrow \mathbb{R}$ is a symmetric periodic function having 1 as smallest period. More precisely, we assume that the function $f$ belongs to the set $\mathcal{F}_{0}=\cup_{\rho>0} \mathcal{F}_{0}(\rho)$ with

$$
\mathcal{F}_{0}(\rho)=\left\{f \in L_{l o c}^{2}: f(x)=f(-x)=f(x+1), \forall x \in \mathbb{R} ;\left|f_{1}\right| \geq \rho\right\},
$$

where we denote by $L_{l o c}^{2}$ is the set of all locally squared integrable functions and by $f_{1}=\int_{-1 / 2}^{1 / 2} f(t) \cos (2 \pi t) d t$.

The goal is to estimate the parameter $\vartheta \in \Theta \subset]-T, T]$ with $T<1 / 4$. As explained in [10], the assumption $T<1 / 4$ is necessary for the identifiability of the parameter $\vartheta$. In this context, the unknown function $f$ is considered as an infinite dimensional nuisance parameter.

The first-order asymptotic properties of estimators in closely related models have been studied in $15,18,31,30$. Note also that the model we have just defined is 
an idealized version of the symmetric location model [33, 23] and the shifted curves model [14].

Let us introduce the "sequence model" equivalent to (1). To do this, for any integer $k \geq 1$, we denote

$$
\begin{aligned}
& x_{k}=\sqrt{2} \int_{-1 / 2}^{1 / 2} \cos (2 \pi k t) d x^{\varepsilon}(t), \\
& x_{k}^{*}=\sqrt{2} \int_{-1 / 2}^{1 / 2} \sin (2 \pi k t) d x^{\varepsilon}(t) .
\end{aligned}
$$

Clearly,

$$
\begin{aligned}
& x_{k}=f_{k} \cos (2 \pi k \vartheta)+\varepsilon \xi_{k}, \\
& x_{k}^{*}=f_{k} \sin (2 \pi k \vartheta)+\varepsilon \xi_{k}^{*},
\end{aligned}
$$

where $f_{k}=\sqrt{2} \int_{-1 / 2}^{1 / 2} \cos (2 \pi k t) f(t) d t$ and $\left(\xi_{k}, \xi_{k}^{*}, k=1,2, \ldots\right)$ are independent standard Gaussian random variables. Note that, the laws of likelihood processes (indexed by $\left.(\vartheta, f) \in \mathbb{R} \times \mathcal{F}_{0}\right)$ of the models (11) and (3) coincide implying thus the equivalence of these models. It is useful to mention here that the Fisher information in the problem of estimating $\vartheta$ with fixed $f$ is

$$
I^{\varepsilon}(f)=\varepsilon^{-2} \int_{-1 / 2}^{1 / 2} f^{\prime}(x)^{2} d x=\varepsilon^{-2} \sum_{k \in \mathbb{N}}(2 \pi k)^{2} f_{k}^{2} .
$$

In this paper, we estimate the parameter $\vartheta$ by a version of the method of profile likelihood maximization (cf. [35, p. 106]), which is also known as penalized maximum likelihood estimator [10. We recall briefly its definition. Write $\mathbf{P}_{\vartheta, f}$ (resp. $\mathbf{E}_{\vartheta, f}$ ) for probability measure (resp. expectation) induced by $x^{\varepsilon}$ on the canonical space $C([-1 / 2,1 / 2])$ equipped with the Wiener measure. As no confusion is possible, we use the same notation in the "sequence model" given by (3). The Radon density $L_{\varepsilon}(\tau, f, \cdot)$ of $\mathbf{P}_{\tau, f}$ with respect to $\mathbf{P}_{0,0}$ is given by

$$
\begin{aligned}
L_{\varepsilon}\left(\tau, f, x^{\varepsilon}\right) & =\exp \left(\varepsilon^{-2} \int_{-1 / 2}^{1 / 2} f(t-\tau) d x^{\varepsilon}(t)-\frac{\varepsilon^{-2}}{2} \int_{-1 / 2}^{1 / 2} f^{2}(t) d t\right) \\
& =\exp \left(\frac{\sqrt{2}}{\varepsilon^{2}} \sum_{k=1}^{\infty} f_{k} \int_{-1 / 2}^{1 / 2} \cos [2 \pi k(t-\tau)] d x^{\varepsilon}(t)-\frac{1}{2 \varepsilon^{2}} \sum_{k=1}^{\infty} f_{k}^{2}\right) .
\end{aligned}
$$

Easy algebra yields

$$
\max _{\left(f_{k}\right)_{k} \in \mathbb{R}^{\mathbb{N}}} L_{\varepsilon}\left(\tau, f, x^{\varepsilon}\right)=\exp \left\{\varepsilon^{-2} \sum_{k=1}^{\infty}\left(\int_{-1 / 2}^{1 / 2} \cos [2 \pi k(t-\tau)] d x^{\varepsilon}(t)\right)^{2}\right\} .
$$

For any $\tau \in \mathbb{R}$, this expression is equal to infinity for almost all paths $x^{\varepsilon}$. Thus, it is necessary to impose some restrictions on the set over which the maximization is done. This is equivalent to considering a penalized profile likelihood. In this paper, we focus on the penalization corresponding to ellipsoids in $\ell^{2}$. More details on this method can be found in [10, we here content ourselves with giving the final definition. 
We call filtering sequence or filter any $h=\left(h_{k}\right)_{k \in \mathbb{N}} \in[0,1]^{\mathbb{N}}$ such that only a finite number of $h_{k}$ are non-zero. Define the functional

$$
\Phi_{\varepsilon}(\tau, h)=\sum_{k=1}^{\infty} h_{k}\left(\int_{-1 / 2}^{1 / 2} \cos [2 \pi k(t-\tau)] d x^{\varepsilon}(t)\right)^{2} .
$$

The PMLE of $\vartheta$ is then $\hat{\vartheta}_{\mathrm{PMLE}}=\arg \max _{\tau} \Phi_{\varepsilon}(\tau, h)$. The role of the sequence $h$ is thus to filter out the irrelevant terms in the right side of (4), that is to assign a value $h_{k}$ close to zero to the terms corresponding to a small signal-to-noise ratio $\left|f_{k}\right| / \varepsilon$.

For deterministic filters $h$, the asymptotic behavior of the estimator $\hat{\vartheta}_{\text {PMLE }}$ is studied in 10. Under some smoothness assumptions on $f$, for a broad choice of filters $h$, $\hat{\vartheta}_{\mathrm{PMLE}}$ is proved to be first-order asymptotic efficient. Moreover, it is shown that the second-order term of its risk expansion is $\varepsilon^{2} R^{\varepsilon}[f, h] /\left\|f^{\prime}\right\|^{4}$, where

$$
R^{\varepsilon}[f, h]=\sum_{k=1}^{\infty}(2 \pi k)^{2}\left[\left(1-h_{k}\right)^{2} f_{k}^{2}+\varepsilon^{2} h_{k}^{2}\right] .
$$

This result suggests to use the filter $h_{o p t}=\arg \min _{h} R^{\varepsilon}[f, h]$ for defining the PMLE of $\vartheta$. However, this minimizer is inapplicable since it depends on $f$. To get rid of this dependence, the minimax approach recommends the utilization of the filter $h_{\mathcal{F}}=\arg \min _{h} \sup _{f \in \mathcal{F}} R^{\varepsilon}[f, h]$. If $\mathcal{F}$ is a ball in a Sobolev space, a solution of this minimization problem is given by the Pinsker filter 27. Although the latter leads to a second-order minimax estimator of $\vartheta[10$, Thm. 2 and 3], it suffers from the well known drawbacks of the minimax theory: the obtained estimator is pessimistic and requires the precise knowledge of the smoothness of the unknown function.

The aim of the present paper is to propose a data-driven filter $\hat{h}$ so that the resulting PMLE of $\vartheta$ circumvents these drawbacks. To be more precise, let us call oracle related to the class of filters $\widehat{\mathcal{H}}$ the value $\arg \min _{h \in \widehat{\mathcal{H}}} R^{\varepsilon}[f, h]$. We cannot use this oracle in our estimating procedure because it depends on the unknown $f$. Nevertheless, the estimator we propose mimics well the behavior of the oracle related to the classe of monotone filters (cf. Section 4.2) for a fixed function $f$ and is second-order sharp adaptive over a broad scale of Sobolev balls.

\section{PMLE BASED ON A DATA-DRIVEN FILTER}

3.1. Local properties of $\Phi_{\varepsilon}(\tau, h)$. Let us introduce some auxiliary notation:

$$
\begin{aligned}
& y_{k}(\tau)=x_{k}(\tau)+i x_{k}^{*}(\tau)=\sqrt{2} \int_{-1 / 2}^{1 / 2} e^{2 i \pi k(t-\tau)} d x^{\varepsilon}(t), \\
& z_{k}(\tau)=\xi_{k}(\tau)+i \xi_{k}^{*}(\tau)=\sqrt{2} \int_{-1 / 2}^{1 / 2} e^{2 i \pi k(t-\tau)} d W(t) .
\end{aligned}
$$

We will write $x_{k}=x_{k}(0), x_{k}^{*}=x_{k}^{*}(0)$ and similarly for $\xi_{k}$ and $\xi_{k}^{*}$. The symmetry of $f$ implies that $x_{k}(\vartheta)=f_{k}+\varepsilon \xi_{k}(\vartheta)$ and $x_{k}^{*}(\vartheta)=\varepsilon \xi_{k}^{*}(\vartheta)$. Moreover, for all $\tau \in \mathbb{R}$, the random variables $\left\{\xi_{i}(\tau), \xi_{i}^{*}(\tau) ; i \in \mathbb{N}\right\}$ are i.i.d. standard Gaussian. Using this 
notation, the functional $\Phi_{\varepsilon}$ can be rewritten as follows

$$
\Phi_{\varepsilon}(\tau, h)=\frac{1}{2} \sum_{k=1}^{\infty} h_{k}\left(x_{k}(\vartheta) \cos [2 \pi k(\tau-\vartheta)]+x_{k}^{*}(\vartheta) \sin [2 \pi k(\tau-\vartheta)]\right)^{2} .
$$

Our aim is to show that under some assumptions on $h$, the function $\Phi_{\varepsilon}(\cdot, h)$ has, with a probability close to one, a local maximum in a neighborhood of $\vartheta$. Note that the derivative of the function $\tau \mapsto \Phi_{\varepsilon}(\tau, h)$ is given by

$$
\begin{aligned}
\Phi_{\varepsilon}^{\prime}(\tau, h)= & -\sum_{k=1}^{\infty} h_{k} \pi k\left(x_{k}(\vartheta)^{2}-x_{k}^{*}(\vartheta)^{2}\right) \sin [4 \pi k(\tau-\vartheta)] \\
& +\sum_{k=1}^{\infty} 2 h_{k} \pi k x_{k}(\vartheta) x_{k}^{*}(\vartheta) \cos [4 \pi k(\tau-\vartheta)] .
\end{aligned}
$$

Proposition 1. Let $\left\|f^{\left(\beta_{*}\right)}\right\|^{2}=\sum_{k}(2 \pi k)^{2 \beta_{*}} f_{k}^{2}<\infty$ for some $\beta_{*}>1$ and set $\hat{\beta}_{*}=\beta_{*} \wedge 1.5, N_{\varepsilon}=\left[\left(\varepsilon^{2} \log \varepsilon^{-5}\right)^{-\frac{1}{2 \beta_{*}+1}}\right]$. Let $h \in[0,1]^{N_{\varepsilon}}$ be a random vector depending on $\left(x_{k}, x_{k}^{*}\right)$ only via $x_{k}^{2}+x_{k}^{* 2}$. For any $\varepsilon<1$, there exists an event $\mathcal{A}_{\varepsilon}$ such that $\mathbf{P}_{\vartheta, f}\left(\mathcal{A}_{\varepsilon}^{c}\right) \leq 2 \varepsilon^{4}$ and on $\mathcal{A}_{\varepsilon} \cap\left\{\sum_{k=1}^{\infty}\left(1-h_{k}\right)(2 \pi k)^{2} f_{k}^{2} \leq\left\|f^{\prime}\right\|^{2} / 4\right\}$, for all $\varepsilon$ verifying

$$
N_{\varepsilon}^{1-\hat{\beta}_{*}} \leq \frac{\left\|f^{\prime}\right\|}{2\left\|f^{\left(\beta_{*}\right)}\right\|+6 \pi},
$$

the function $\tau \mapsto \Phi_{\varepsilon}(\tau, h)$ is strictly concave and admits a unique maximum $\hat{\vartheta}_{\varepsilon}$ in the interval $\left[\vartheta-\left(4 \pi N_{\varepsilon}\right)^{-1}, \vartheta+\left(4 \pi N_{\varepsilon}\right)^{-1}\right]$, satisfying

$$
\left|\hat{\vartheta}_{\varepsilon}-\vartheta\right| \leq 8 \varepsilon \sqrt{\log \varepsilon^{-5}}\left\|f^{\prime}\right\|^{-1} .
$$

Proof. Set $\Theta_{\varepsilon}=\left[\vartheta-\left(4 \pi N_{\varepsilon}\right)^{-1}, \vartheta+\left(4 \pi N_{\varepsilon}\right)^{-1}\right]$. Assume that (7) is fulfilled and $\sum_{k=1}^{\infty}\left(1-h_{k}\right)(2 \pi k)^{2} f_{k}^{2} \leq\left\|f^{\prime}\right\|^{2} / 4$. On the one hand, the first inequality of Proposition 4 (see Section 5.2 below) implies that there exists an event $\mathcal{A}_{\varepsilon}$, such that on this event

$$
\Phi_{\varepsilon}^{\prime \prime}(\tau, h) \leq-\left\|f^{\prime}\right\|^{2}+\sum_{k=1}^{\infty}\left(1-h_{k}\right)(2 \pi k)^{2} f_{k}^{2}+\left\|f^{\prime}\right\|^{2} / 4 \leq-\left\|f^{\prime}\right\|^{2} / 2,
$$

for all $\tau \in \Theta_{\varepsilon}$ and for $\varepsilon$ small enough. Therefore, $\Phi_{\varepsilon}(\cdot, h)$ is strictly concave. On the other hand, the second inequality of Proposition 4 implies that

$$
\begin{aligned}
\frac{\Phi_{\varepsilon}^{\prime}(\tau, h)}{\tau-\vartheta} & \leq-\frac{\left\|f^{\prime}\right\|^{2}}{2}+\frac{2 \varepsilon\left(\left\|f^{\prime}\right\| \sqrt{\log \varepsilon^{-5}}+2 \pi N_{\varepsilon}^{1-\hat{\beta}_{*}}\right)}{|\tau-\vartheta|} \\
& \leq-\frac{\left\|f^{\prime}\right\|^{2}}{2}+\frac{4 \varepsilon \sqrt{\log \varepsilon^{-5}}\left\|f^{\prime}\right\|}{|\tau-\vartheta|}
\end{aligned}
$$

for sufficiently small values of $\varepsilon$. Therefore, $\pm \Phi_{\varepsilon}^{\prime}\left(\vartheta \pm\left(4 \pi N_{\varepsilon}\right)^{-1}, h\right)<0$, which guarantees that the maximum $\hat{\vartheta}_{\varepsilon}$ of $\Phi_{\varepsilon}(\cdot, h)$ is attained in the interior of $\Theta_{\varepsilon}$ and $\Phi_{\varepsilon}^{\prime}\left(\hat{\vartheta}_{\varepsilon}, h\right)=0$. Applying (9) to $\tau=\hat{\vartheta}_{\varepsilon}$ we get (8)).

Remark 1. The choice $N_{\varepsilon}=\left[\left(\varepsilon^{2} \log \varepsilon^{-5}\right)^{-\frac{1}{2 \beta_{*}+1}}\right]$ has a simple interpretation. If $f_{k}^{2} \leq \varepsilon^{2}$ for some $k \in \mathbb{N}$, then the $k$ th observation in (3) is not relevant for estimating 
the parameter $\vartheta$. Let $\mathcal{K}=\left\{k \in \mathbb{N}: f_{k}^{2}>\varepsilon^{2}\right\}$ and $K=\# \mathcal{K}$. Then

$$
\sum_{k \in \mathcal{K}}(2 \pi k)^{2 \beta_{*}} f_{k}^{2} \geq \varepsilon^{2} \sum_{k \in \mathcal{K}}(2 \pi k)^{2 \beta_{*}} \geq \varepsilon^{2} \sum_{k=1}^{K}(2 \pi k)^{2 \beta_{*}} \geq \frac{\varepsilon^{2}(2 \pi)^{2 \beta_{*}} K^{2 \beta_{*}+1}}{2 \beta_{*}+1} .
$$

Thus, the number $K$ of Fourier coefficients $f_{k}$ larger than $\varepsilon$ is at most $O\left(\varepsilon^{-\frac{2}{2 \beta_{*}+1}}\right)$. Thus, for $\varepsilon$ small enough, all observations relevant for estimating the parameter $\vartheta$ lie in $\left\{y_{1}, \ldots, y_{N}\right\}$.

Remark 2. In [10], the estimator $\hat{\vartheta}_{\mathrm{PMLE}}$ is defined as the maximizer of $\Phi_{\varepsilon}(\cdot, h)$ over the whole interval $\Theta$. Instead, we define it as the local maximizer in the neighborhood of a preliminary estimator. This modification is explained by the fact that the function $\tau \mapsto \Phi_{\varepsilon}(\tau, h)$ is only locally concave when $\beta_{*}<2$. Furthermore, the computation of the local minimum is faster than the computation of the global minimum.

Remark 3. Elaborating on the arguments of Proposition 1 it can be shown that Thm. 1 from [10 remains true for $\beta>1$, provided that $\vartheta_{\mathrm{PMLE}}$ is defined as the local maximizer of $\Phi_{\varepsilon}(\cdot, h)$ and condition B2 is replaced by $h_{k}=0$ for any $k>N_{\varepsilon}$.

3.2. Blockwise constant Stein filter with penalization. Let $J$ be a positive integer and $\kappa_{1}, \ldots, \kappa_{J+1} \in\left\{1, \ldots, N_{\varepsilon}\right\}$ be a strictly increasing sequence such that $\kappa_{1}=1$. Set $B_{j}=\left\{k \in \mathbb{N}: \kappa_{j} \leq k<\kappa_{j+1}\right\}$. Let $\mathcal{H}^{*}(B)$ be the set of all filters $h \in[0,1]^{N_{\varepsilon}}$ that are constant on the blocks $B=\left\{B_{j}\right\}_{j=1}^{J}$ :

$$
h \in \mathcal{H}^{*}(B) \quad \Longleftrightarrow \quad h_{k}=h_{k^{\prime}}, \quad \forall k, k^{\prime} \in B_{j} .
$$

An oracle knowing the function $f$, would choose the best possible filter $h^{*} \in \mathcal{H}^{*}(B)$ by minimizing $R^{\varepsilon}[f, h]$ over $\mathcal{H}^{*}(B)$. We call $h^{*}$ oracle choice of filter or simply oracle. Simple computations show that

$$
h_{k}^{*}=\frac{\left\|f^{\prime}\right\|_{(j)}^{2}}{\left\|f^{\prime}\right\|_{(j)}^{2}+\varepsilon^{2} \sigma_{j}^{2}}, \quad k \in B_{j}, \quad j=1, \ldots, J,
$$

where $\left\|f^{\prime}\right\|_{(j)}^{2}=\sum_{k \in B_{j}}(2 \pi k)^{2} f_{k}^{2}$ and $\sigma_{j}^{2}=\sum_{k \in B_{j}}(2 \pi k)^{2}$.

Since the oracle $h^{*}$ depends on $f$, we replace it by a suitable estimator. Let us define $R_{j}^{\varepsilon}[f, a]=(1-a)^{2}\left\|f^{\prime}\right\|_{(j)}^{2}+\varepsilon^{2} a^{2} \sigma_{j}^{2}$ so that $R^{\varepsilon}[f, h]=\sum_{j} R_{j}^{\varepsilon}\left[f, h_{\kappa_{j}}\right]+$ $\sum_{k>N_{\varepsilon}}(2 \pi k)^{2} f_{k}^{2}$. Then $h_{\kappa_{j}}^{*}$ is the minimizer of $R_{j}^{\varepsilon}[f, a]$ over $a \in \mathbb{R}$. Define $\left\|y^{\prime}\right\|_{(j)}^{2}=\sum_{k \in B_{j}}(2 \pi k)^{2}\left|y_{k}\right|^{2}$ and $y_{k}=x_{k}+i x_{k}^{*}$. For small values of $h_{\kappa_{j}}^{*}$, the minimizer of

$$
\hat{R}_{j}^{\varepsilon}[a]:=(1-a)^{2}\left(\left\|y^{\prime}\right\|_{(j)}^{2}-2 \varepsilon^{2} \sigma_{j}^{2}\right)_{+}+\varepsilon^{2} a^{2} \sigma_{j}^{2},
$$

which is an estimator of $R_{j}^{\varepsilon}[f, a]$, can be large with respect to $h_{\kappa_{j}}^{*}$. To avoid such a configuration, we penalize large values of $a$ and define the estimator $\hat{h}^{J S}$ of $h^{*}$ as the minimum over $[0,1]$ of the function:

$$
a \mapsto \hat{R}_{j}^{\varepsilon}[a]+2 \varphi_{j} \varepsilon^{2} \sigma_{j}^{2} a,
$$

where $\varphi_{j}>0$ is a factor of penalization tending to zero as $\varepsilon \rightarrow 0$. This leads us to the penalized Stein filter

$$
\hat{h}_{k}^{J S}=\left(1-\frac{\varepsilon^{2} \sigma_{j}^{2}\left(1+\varphi_{j}\right)}{\left(\left\|y^{\prime}\right\|_{(j)}^{2}-2 \varepsilon^{2} \sigma_{j}^{2}\right)_{+}+\varepsilon^{2} \sigma_{j}^{2}}\right)_{+}, \quad \forall k \in B_{j} .
$$


3.3. Weakly geometrically increasing blocks. The aim of this section is to propose a concrete scheme for defining the blockwise constant data-driven filter. We use the weakly geometrically increasing blocks introduced by Cavalier and Tsybakov [8, 9]. These blocks have the advantage of being simple (the construction is driven by only one parameter) and of having good approximation properties with respect to the class of monotone filters [9, Lemma 1].

Let $\nu=\nu_{\varepsilon}$ be a positive integer that increases as $\varepsilon$ decreases. Set $\rho_{\varepsilon}=\nu_{\varepsilon}^{-1 / 3}$ and define

$$
\kappa_{j}= \begin{cases}\left(1+\nu_{\varepsilon}\right)^{j-1}, & j=1,2, \\ \kappa_{j-1}+\left\lfloor\nu_{\varepsilon} \rho_{\varepsilon}\left(1+\rho_{\varepsilon}\right)^{j-2}\right\rfloor, & j=3,4, \ldots,\end{cases}
$$

where $\lfloor x\rfloor$ stands for the largest integer strictly smaller than $x$. Let $J$ be the smallest integer $j$ such that $\kappa_{j} \geq N_{\varepsilon}+1$. We redefine $\kappa_{J+1}=N_{\varepsilon}+1$ and set $B_{j}=\left\{\kappa_{j}, \ldots, \kappa_{j+1}-1\right\}$ for all $j=1, \ldots, J$.

3.4. Brief description of the procedure. The outlined scheme can be implemented as follows.

1. Choose a real number $\beta_{*}>1$ and set $N_{\varepsilon}=5 \vee\left[\left(\varepsilon^{2} \log \varepsilon^{-5}\right)^{-\frac{1}{2 \beta_{*}+1}}\right], \nu_{\varepsilon}=$ $\left[e^{\sqrt{\log N_{\varepsilon}}}\right]$ and $\rho_{\varepsilon}=\nu_{\varepsilon}^{-1 / 3}$.

2. Define the sequence $\left(\kappa_{j}\right)_{j}$ by (11).

3. Set $\varphi_{j}=\sqrt{24 \log \varepsilon^{-5} /\left(\kappa_{j+1}-\kappa_{j}\right)}, \sigma_{j}^{2}=\sum_{\kappa_{j} \leq k<\kappa_{j+1}}(2 \pi k)^{2}$ and define the data-dependent filter $\hat{h}^{J S}$ by (10).

4. Compute the preliminary estimator $\bar{\vartheta}_{\varepsilon}$ (cf. Section 5) and set $\bar{\Theta}_{\varepsilon}=\left[\bar{\vartheta}_{\varepsilon}-\right.$ $\left.\delta_{\varepsilon}, \bar{\vartheta}_{\varepsilon}+\delta_{\varepsilon}\right]$ with $\delta_{\varepsilon}=\varepsilon \log \left(\varepsilon^{-2}\right)$.

5. Define $\hat{\vartheta}_{\varepsilon}$ as the minimum in $\bar{\Theta}_{\varepsilon}$ of $\Phi_{\varepsilon}\left(\cdot, \hat{h}^{J S}\right)$ (see (5)).

Note that the only "free" parameter in this procedure is $\beta_{*}$. In practice, if no information on the regularity of $f$ is available, it appears plausible to assume that $f$ has Sobolev smoothness $\beta_{*}=2$.

\section{MAIN RESUlTS}

4.1. Comparison with the blockwise constant oracle. In this section, $\hat{h}^{J S}$ denotes the blockwise constant filter defined by (10), $\varphi_{j}$ is the penalization we use on the block $B_{j}$ and $\varphi_{\varepsilon}=\max _{j} \varphi_{j}$. We emphasize that in this section no condition on the blocks $B_{j}$ is required. Let $T_{j}$ be the length of the block $B_{j}$ and $T_{\varepsilon}=\inf _{j} T_{j}$. The oracle choice of $h$ in the class $\mathcal{H}^{*}(B)$ of all filters constant on the blocks $B=\left\{B_{j}\right\}_{j}$ is denoted by $h^{*}$. Define

$$
\hat{\vartheta}_{\varepsilon}^{J S}=\underset{\tau \in \bar{\Theta}_{\varepsilon}}{\arg \max } \Phi_{\varepsilon}\left(\tau, \hat{h}^{J S}\right),
$$

where $\bar{\Theta}_{\varepsilon}=\left[\bar{\vartheta}_{\varepsilon}-\delta_{\varepsilon}, \bar{\vartheta}_{\varepsilon}+\delta_{\varepsilon}\right]$ and $\bar{\vartheta}_{\varepsilon}$ is a rate optimal initial estimator of $\vartheta$ (cf. Section 5.11). Introduce the functional class

$$
\mathcal{F}\left(\beta_{*}, L_{*}, \rho\right)=\left\{f \in \mathcal{F}_{0}(\rho):\left\|f^{\left(\beta_{*}\right)}\right\| \leq L_{*}\right\},
$$

where $\beta_{*}>1, \rho>0, L_{*}>0$ are some constants. 
Theorem 1. Let $\hat{\vartheta}_{\varepsilon}^{J S}$ be defined by (12) with blocks $B_{j}$ verifying $\log \varepsilon^{-1}=o\left(T_{\varepsilon}\right)$ as $\varepsilon \rightarrow 0$. If the penalty $\varphi_{j}$ is equal to $\sqrt{24 T_{j}^{-1} \log \varepsilon^{-5}}$, then

$$
\varepsilon^{-2}\left\|f^{\prime}\right\|^{2} \mathbf{E}_{\vartheta, f}\left[\left(\hat{\vartheta}_{\varepsilon}^{J S}-\vartheta\right)^{2}\right] \leq 1+\left(1+\alpha_{\varepsilon}\right) \frac{R^{\varepsilon}\left[f, h^{*}\right]}{\left\|f^{\prime}\right\|^{2}},
$$

where $\alpha_{\varepsilon} \rightarrow 0$ as $\varepsilon \rightarrow 0$ uniformly in $f \in \mathcal{F}\left(\beta_{*}, L_{*}, \rho\right)$.

Remark 4. If the block $B_{j}$ is large, then more observations $\left(x_{k}, x_{k}^{*}\right)$ are used for estimating the value of the oracle $h_{\kappa_{j}}^{*}$. Hence, it is natural to expect that $\alpha_{\varepsilon}$ decreases as $T_{\varepsilon}$ increases. A thorough inspection of the proof allows to describe this feature with the help of the order relation $\alpha_{\varepsilon}^{2} \asymp T_{\varepsilon}^{-1} \log \varepsilon^{-1}$.

Proof. Let us denote $\mathcal{E}=\left\{\hat{h}_{k}^{J S} \in\left[h_{k}^{*}-\sqrt{8} \varphi_{j(k)}\left(1-h_{k}^{*}\right), h_{k}^{*}\right], \forall k=1, \ldots, N_{\varepsilon}\right\}$ and $\tilde{h}=\hat{h}^{J S} \mathbb{1}_{\mathcal{E}}$, where $j(k)$ is the number of the block containing $k$. Lemma 5 implies that $\mathbf{P}_{\vartheta, f}\left(\mathcal{E}^{c}\right) \leq 2 \varepsilon^{4}$. For $\varepsilon$ small enough, we have $\left(1-\tilde{h}_{k}\right) \mathbb{1}_{\mathcal{E}} \leq 2\left(1-h_{k}^{*}\right)$ and the inequality $\sum_{k}\left(1-\hat{h}_{k}\right)(2 \pi k)^{2} f_{k}^{2} \leq\left\|f^{\prime}\right\|^{2} / 4$ is fulfilled on $\mathcal{E}$.

By virtue of Proposition 3 , for sufficiently small values of $\varepsilon$, the event $\mathcal{A}_{0}=\left\{\mid \bar{\vartheta}_{\varepsilon}-\right.$ $\left.\vartheta \mid \leq\left(\frac{1}{4 \pi N_{\varepsilon}}-\delta_{\varepsilon}\right) \wedge\left(\delta_{\varepsilon}-\frac{8 \varepsilon \sqrt{\log \varepsilon^{-5}}}{\left\|f^{\prime}\right\|}\right)\right\}$ verifies $\mathbf{P}_{\vartheta, f}\left(\mathcal{A}_{0}^{c}\right)=O\left(\varepsilon^{4}\right)$. It can be checked that on $\mathcal{A}_{0},\left[\vartheta \pm \frac{8 \varepsilon \sqrt{\log \varepsilon^{-5}}}{\left\|f^{\prime}\right\|}\right] \subset\left[\bar{\vartheta}_{\varepsilon} \pm \delta_{\varepsilon}\right] \subset\left[\vartheta \pm \frac{1}{4 \pi N_{\varepsilon}}\right]$, where we have used the notation $[\vartheta \pm \delta]:=[\vartheta-\delta, \vartheta+\delta]$.

According to Proposition [1] on the event $\mathcal{A}_{1}=\mathcal{A}_{0} \cap \mathcal{A}_{\varepsilon} \cap \mathcal{E}$, the function $\Phi_{\varepsilon}(\cdot, \hat{h})$ is strictly concave and has a unique maximum in $\left[\bar{\vartheta}_{\varepsilon} \pm \delta_{\varepsilon}\right]$. Hence the estimator $\hat{\vartheta}_{\varepsilon}^{J S}$ verifies $\Phi_{\varepsilon}^{\prime}\left(\hat{\vartheta}_{\varepsilon}^{J S}, \tilde{h}\right)=0$ on the event $\mathcal{A}_{1}$. By Taylor's formula, there exists a point $\tilde{\vartheta} \in\left[\vartheta, \hat{\vartheta}_{\varepsilon}^{J S}\right]$ such that

$$
0=\Phi_{\varepsilon}^{\prime}\left(\hat{\vartheta}_{\varepsilon}^{J S}, \tilde{h}\right)=\Phi_{\varepsilon}^{\prime}(\vartheta, \tilde{h})+\left(\hat{\vartheta}_{\varepsilon}^{J S}-\vartheta\right) \Phi_{\varepsilon}^{\prime \prime}(\vartheta, \tilde{h})+\frac{\left(\hat{\vartheta}_{\varepsilon}^{J S}-\vartheta\right)^{2}}{2} \Phi_{\varepsilon}^{\prime \prime \prime}(\tilde{\vartheta}, \tilde{h}) .
$$

Therefore, on $\mathcal{A}_{1}$,

$$
\hat{\vartheta}_{\varepsilon}^{J S}-\vartheta=-\frac{\Phi_{\varepsilon}^{\prime}(\vartheta, \tilde{h})}{\Phi_{\varepsilon}^{\prime \prime}(\vartheta, \tilde{h})+\frac{1}{2}\left(\hat{\vartheta}_{\varepsilon}^{J S}-\vartheta\right) \Phi_{\varepsilon}^{\prime \prime \prime}(\tilde{\vartheta}, \tilde{h})} .
$$

Using (6), one checks that

$$
\Phi_{\varepsilon}^{\prime \prime}(\vartheta, \tilde{h})=-\sum_{k=1}^{\infty} \tilde{h}_{k}(2 \pi k)^{2}\left[\left(f_{k}+\varepsilon \xi_{k}(\vartheta)\right)^{2}-\varepsilon^{2} \xi_{k}^{*}(\vartheta)^{2}\right] .
$$

In the sequel, we write $\xi_{k}, \xi_{k}^{*}$ instead of $\xi_{k}(\vartheta), \xi_{k}^{*}(\vartheta)$. On the one hand, Lemmas 6. 7 (with $x^{2}=\log \varepsilon^{-5}$ ) and 8 combined with (22) imply that, on an event $\mathcal{A}_{2}$ of probability higher than $1-4 \varepsilon^{4}$, we have

$$
\begin{aligned}
\Phi_{\varepsilon}^{\prime \prime}(\vartheta, \tilde{h}) & =-\left\|f^{\prime}\right\|^{2}+\sum_{k=1}^{\infty}\left(1-h_{k}^{*}\right)(2 \pi k)^{2} f_{k}^{2}-2 \varepsilon \sum_{k=1}^{N_{\varepsilon}} h_{k}^{*}(2 \pi k)^{2} f_{k} \xi_{k}+o\left(R^{\varepsilon}\right) \\
& =-\left\|f^{\prime}\right\|^{2}\left(1-\left\|f^{\prime}\right\|^{-2} R^{\varepsilon}\left[f, h^{*}\right]-\zeta-o\left(R^{\varepsilon}\left[f, h^{*}\right]\right)\right),
\end{aligned}
$$


where $\zeta=2 \varepsilon\left\|f^{\prime}\right\|^{-2} \sum_{k} h_{k}^{*}(2 \pi k)^{2} f_{k} \xi_{k}$ is a zero mean Gaussian random variable. By virtue of (16), its variance verifies

$$
4 \varepsilon^{2} \sum_{k=1}^{N_{\varepsilon}} h_{k}^{* 2}(2 \pi k)^{4} f_{k}^{2} \leq \frac{12 \varepsilon^{2}}{\min _{j} T_{j}} \sum_{j=1}^{J} h_{\kappa_{j}}^{* 2} \sigma_{j}^{2}\left\|f^{\prime}\right\|_{(j)}^{2} \leq \frac{12\left\|f^{\prime}\right\|^{2} R^{\varepsilon}\left[f, h^{*}\right]}{T_{\varepsilon}} .
$$

Therefore, by Rosenthal's inequality, $\mathbf{E}_{\vartheta, f}\left[\zeta^{2 p}\right]^{1 / p}=o\left(R^{\varepsilon}\left[f, h^{*}\right]\right)$ for any $p>0$. On the other hand, in view of Lemma 9, there is an event $\mathcal{A}_{3}$ such that $\mathbf{P}_{\vartheta, f}\left(\mathcal{A}_{3}^{c}\right)=$ $O\left(\varepsilon^{4}\right)$ and

$$
\left(\hat{\vartheta}_{\varepsilon}^{J S}-\vartheta\right) \Phi_{\varepsilon}^{\prime \prime \prime}(\tilde{\vartheta}, \tilde{h})=o\left(R^{\varepsilon}\right)
$$

on this event. Using the inequality $(1-x)^{-2} \leq 1+2 x+16 x^{2}$ for all $x \in[-1 / 2,1 / 2]$, we get

$$
\begin{aligned}
\left\|f^{\prime}\right\|^{4}\left(\hat{\vartheta}_{\varepsilon}^{J S}-\vartheta\right)^{2} & =\frac{\Phi_{\varepsilon}^{\prime}(\vartheta, \tilde{h})^{2}}{\left(1-\left\|f^{\prime}\right\|^{-2} R^{\varepsilon}\left[f, h^{*}\right]-\zeta-o\left(R^{\varepsilon}\right)\right)^{2}} \\
& \leq \Phi_{\varepsilon}^{\prime}(\vartheta, \tilde{h})^{2}\left(1+\frac{2 R^{\varepsilon}\left[f, h^{*}\right]}{\left\|f^{\prime}\right\|^{2}}+2 \zeta+o\left(R^{\varepsilon}\right)\right)+o\left(\varepsilon^{2} R^{\varepsilon}\right),
\end{aligned}
$$

on the event $\mathcal{A}_{4}=\mathcal{A}_{1} \cap \mathcal{A}_{2} \cap \mathcal{A}_{3}$. Using Lemma 3, we infer that

$$
\begin{aligned}
\mathbf{E}_{\vartheta, f}\left[\Phi_{\varepsilon}^{\prime}(\vartheta, \tilde{h})^{2}\right] & \leq \varepsilon^{2}\left\|f^{\prime}\right\|^{2}+\varepsilon^{2} \sum_{k=1}^{\infty}(2 \pi k)^{2}\left[\left(h_{k}^{* 2}-1\right) f_{k}^{2}+h_{k}^{* 2} \varepsilon^{2}\right] \\
& =\varepsilon^{2}\left(\left\|f^{\prime}\right\|^{2}-R^{\varepsilon}\left[f, h^{*}\right]\right) .
\end{aligned}
$$

Combining these relations with Lemmas 10 and 11, we get an event $\mathcal{A}$ such that $\mathbf{P}_{\vartheta, f}\left(\mathcal{A}^{c}\right)=O\left(\varepsilon^{4}\right)$ and

$$
\begin{aligned}
\left\|f^{\prime}\right\|^{4} \mathbf{E}_{\vartheta, f}\left[\left(\hat{\vartheta}_{\varepsilon}^{J S}-\vartheta\right)^{2} \mathbb{1}_{\mathcal{A}}\right] & \leq \mathbf{E}_{\vartheta, f}\left[\Phi_{\varepsilon}^{\prime}(\vartheta, \tilde{h})^{2}\right]\left(1+\frac{2 R^{\varepsilon}\left[f, h^{*}\right]}{\left\|f^{\prime}\right\|^{2}}\right)+o\left(\varepsilon^{2} R^{\varepsilon}\left[f, h^{*}\right]\right) \\
& \leq \varepsilon^{2}\left\|f^{\prime}\right\|^{2}+\varepsilon^{2} R^{\varepsilon}\left[f, h^{*}\right]+o\left(\varepsilon^{2} R^{\varepsilon}\left[f, h^{*}\right]\right) .
\end{aligned}
$$

Since $\left|\hat{\vartheta}_{\varepsilon}^{J S}-\vartheta\right| \leq 1$, we have $\mathbf{E}_{\vartheta, f}\left[\left(\hat{\vartheta}_{\varepsilon}^{J S}-\vartheta\right)^{2} \mathbb{1}_{\mathcal{A}^{c}}\right] \leq \mathbf{P}_{\vartheta, f}\left(\mathcal{A}^{c}\right)=O\left(\varepsilon^{4}\right)$. In view of (23) $), \varepsilon^{2}=o\left(R^{\varepsilon}\left[f, h^{*}\right]\right)$. Therefore $\mathbf{E}_{\vartheta, f}\left[\left(\hat{\vartheta}_{\varepsilon}^{J S}-\vartheta\right)^{2} \mathbb{1}_{\mathcal{A}^{c}}\right]=O\left(\varepsilon^{4}\right)=o\left(\varepsilon^{2} R^{\varepsilon}\left[f, h^{*}\right]\right)$ and the assertion of the theorem follows.

4.2. Comparison with the monotone oracle. Now we consider the class $\mathcal{H}_{\text {mon }}$ of filters having decreasing components, that is

$$
\mathcal{H}_{\text {mon }}=\left\{h \in[0,1]^{N_{\varepsilon}}: h_{k} \geq h_{k+1}, 1 \leq k \leq N_{\varepsilon}-1\right\} .
$$

The class $\mathcal{H}_{\text {mon }}$ is of high interest in statistics because it contains the most common filters such as the projection filter, the Pinsker filter, the Tikhonov or smoothing spline filter and so forth.

Proposition 2. Set $\gamma_{\varepsilon}=\max _{1 \leq j \leq J-1}\left(\sigma_{j+1}^{2} / \sigma_{j}^{2}\right)$. Then

$$
R^{\varepsilon}\left[f, h^{*}\right] \leq \gamma_{\varepsilon} \inf _{h \in \mathcal{H}_{\text {mon }}} R^{\varepsilon}[f, h]+\varepsilon^{2} \sigma_{1}^{2} .
$$

A more general version of this result is Lemma 1 in $[9$. Since the proof in our setting is simple, we give it below. 
Proof. Let $h$ be a filter from $\mathcal{H}_{\text {mon }}$. Define $\bar{h}$ by $\bar{h}_{k}=h_{\kappa_{j}}$ if $k \in B_{j}$ for some $j$ and $\bar{h}_{k}=0$ if $k>N_{\varepsilon}$. Since the components of $h$ are decreasing, we have $1-\bar{h}_{k} \leq 1-h_{k}$ and therefore

$$
R^{\varepsilon}[f, \bar{h}] \leq \sum_{k=1}^{N_{\varepsilon}}\left(1-h_{k}\right)^{2}(2 \pi k)^{2} f_{k}^{2}+\varepsilon^{2} \sum_{j=1}^{J} h_{\kappa_{j}}^{2} \sigma_{j}^{2} .
$$

Again by monotonicity of $h$, we have $h_{\kappa_{j}} \leq h_{k}$ for all $k \in B_{j-1}$. Hence,

$$
\sum_{j=2}^{J} h_{\kappa_{j}}^{2} \sigma_{j}^{2} \leq \gamma_{\varepsilon} \sum_{j=2}^{J} h_{\kappa_{j}}^{2} \sigma_{j-1}^{2} \leq \gamma_{\varepsilon} \sum_{k=1}^{N_{\varepsilon}} h_{k}^{2}(2 \pi k)^{2} .
$$

Combining this inequality with (14) and bounding $h_{1}$ by 1 , we get $R^{\varepsilon}[f, \bar{h}] \leq$ $\gamma_{\varepsilon} R^{\varepsilon}[f, h]+\sigma_{1}^{2}$. Since $\bar{h} \in \mathcal{H}^{*}$ and $h^{*}$ minimizes $R^{\varepsilon}[f, h]$ over all $h \in \mathcal{H}^{*}$, we have $R^{\varepsilon}\left[f, h^{*}\right] \leq \gamma_{\varepsilon} R^{\varepsilon}[f, h]+\varepsilon^{2} \sigma_{1}^{2}$. This inequality holds for every $h \in \mathcal{H}_{\text {mon }}$, therefore the assertion of the proposition follows.

Combining this proposition with Theorem 1 we get the following result.

Corollary 1. Assume that the conditions of Theorem 1 are fulfilled, then

$$
\varepsilon^{-2}\left\|f^{\prime}\right\|^{2} \mathbf{E}_{\vartheta, f}\left[\left(\hat{\vartheta}_{\varepsilon}^{J S}-\vartheta\right)^{2}\right] \leq 1+\gamma_{\varepsilon}\left(1+\alpha_{\varepsilon}\right) \frac{\min _{h \in \mathcal{H}_{\text {mon }}} R^{\varepsilon}[f, h]}{\left\|f^{\prime}\right\|^{2}},
$$

where $\alpha_{\varepsilon} \rightarrow 0$ as $\varepsilon \rightarrow 0$ uniformly in $f \in \mathcal{F}\left(\beta_{*}, L_{*}, \rho\right)$.

Remark 5. For the blocks defined by (11), we have $T_{\varepsilon}=\nu_{\varepsilon} \rho_{\varepsilon}\left(1+\rho_{\varepsilon}\right), \sigma_{1}^{2} \leq$ $4 \pi^{2} \nu_{\varepsilon}^{3}$ and $-\nu_{\varepsilon} \rho_{\varepsilon}+\nu_{\varepsilon}\left(1+\rho_{\varepsilon}\right)^{j} \leq \kappa_{j+1} \leq 1+\nu_{\varepsilon}\left(1+\rho_{\varepsilon}\right)^{j}$. One also checks that $\gamma_{\varepsilon}=\max _{j} \sigma_{j+1}^{2} / \sigma_{j}^{2}$ is asymptotically equivalent to $\left(1+\rho_{\varepsilon}\right)^{3} \sim 1+3 \rho_{\varepsilon}$ as $\varepsilon \rightarrow 0$. Therefore the factor in the oracle inequality of Corollary 1 is of order $\left(1+3 \rho_{\varepsilon}+\alpha_{\varepsilon}\right)$. We have already mentioned that $\alpha_{\varepsilon}^{2}=O\left(T_{\varepsilon}^{-1} \log \varepsilon^{-1}\right)$. The trade-off between $\alpha_{\varepsilon}$ and $\rho_{\varepsilon}$ leads us to $\rho_{\varepsilon} \asymp \nu_{\varepsilon}^{-1 / 3}$. This clarifies our choice of $\rho_{\varepsilon}$ slightly differing from the one of 9 .

Remark 6. In $[8,28,34$ the weakly geometrically increasing blocks are defined by $T_{j}=\left\lfloor\nu(1+\rho)^{j-1}\right\rfloor$. This type of blocks does not lead to a sharp oracle inequality in our case, since we need not only $\max \left(T_{j+1} / T_{j}\right) \rightarrow 1$, but also $\max \left(\kappa_{j+1} / \kappa_{j}\right) \rightarrow 1$ as $\varepsilon \rightarrow 0$.

4.3. Second-order minimax sharp adaptation. To complete the theoretical analysis, we show below that the estimator $\hat{\vartheta}_{\varepsilon}^{J S}$ corresponding to the blocks (11) enjoys minimax properties over a large scale of Sobolev balls. Assume that $\bar{f} \in$ $\mathcal{F}\left(\beta^{*}, L^{*}, \rho\right)$ and define

$$
\mathcal{F}_{\delta, \beta, L}(\bar{f})=\left\{f=\bar{f}+v:\|v\| \leq \delta,\left\|v^{(\beta)}\right\| \leq L\right\} .
$$

Theorem 2. Assume that $\nu_{\varepsilon}$ verifies $\varepsilon^{\frac{2}{2 \beta+1}} \nu_{\varepsilon} \rightarrow 0$ as $\varepsilon \rightarrow 0$ and the conditions of Theorem 1 are fulfilled. If $\delta=\delta_{\varepsilon}$ tends to zero as $\varepsilon \rightarrow 0$ and $\bar{f} \in \mathcal{F}\left(\beta^{*}, L^{*}, \rho\right)$ with $\beta^{*}>\beta \geq \beta_{*}$, then the estimator $\hat{\vartheta}_{\varepsilon}^{J S}$ defined in Section 3.4 satisfies

$$
\sup _{\vartheta \in \Theta, f \in \mathcal{F}_{\delta, \beta, L}(\bar{f})} \varepsilon^{-2}\left\|f^{\prime}\right\|^{2} \mathbf{E}_{\vartheta, f}\left[\left(\hat{\vartheta}_{\varepsilon}^{J S}-\vartheta\right)^{2}\right] \leq 1+(1+o(1)) \frac{C(\beta, L) \varepsilon^{\frac{4 \beta-4}{2 \beta+1}}}{\left\|\bar{f}^{\prime}\right\|^{2}},
$$


when $\varepsilon \rightarrow 0$, with $C(\beta, L)=\frac{1}{3}\left(\frac{\beta-1}{2 \pi(\beta+2)}\right)^{\frac{2 \beta-2}{2 \beta+1}}(L(2 \beta+1))^{\frac{3}{2 \beta+1}}$. Moreover, the following lower bound holds:

$$
\inf _{\tilde{\vartheta}_{\varepsilon}} \sup _{\vartheta \in \Theta, f \in \mathcal{F}_{\delta, \beta, L}(\bar{f})} \varepsilon^{-2}\left\|f^{\prime}\right\|^{2} \mathbf{E}_{\vartheta, f}\left[\left(\tilde{\vartheta}_{\varepsilon}-\vartheta\right)^{2}\right] \geq 1+(1+o(1)) \frac{C(\beta, L) \varepsilon^{\frac{4 \beta-4}{2 \beta+1}}}{\left\|\bar{f}^{\prime}\right\|^{2}},
$$

where the inf is taken over all possible estimators $\tilde{\vartheta}_{\varepsilon}$.

Proof. According to Lemma 12, there exists a filter $\lambda^{*} \in \mathcal{H}_{\text {mon }}$ such that

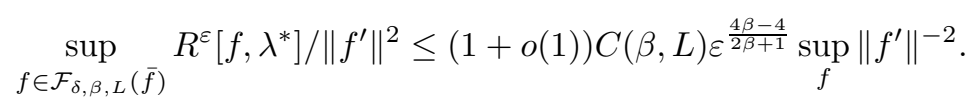

Since $\delta_{\varepsilon} \rightarrow 0$ as $\varepsilon \rightarrow 0$, we have $\sup _{f \in \mathcal{F}_{\delta, \beta, L}(\bar{f})}\left\|f^{\prime}\right\|^{-2}=(1+o(1))\left\|\bar{f}^{\prime}\right\|^{-2}$. Combining this result with Corollary 1 we get the first inequality. The second inequality is Theorem 2 of [10. Although the latter is stated for $\beta \geq 2$, the inspection of its proof shows that the same claim is true for any $\beta>1$.

\section{Preliminary ESTIMATOR AND TECHNiCAL LEMMAS}

5.1. Preliminary estimator. Having the observation $\left(x^{\varepsilon}(t),|t| \leq 1 / 2\right)$, we can compute $x_{1}, x_{1}^{*}$ by (2). Then we have $x_{1}=f_{1} \cos (2 \pi \vartheta)+\varepsilon \xi_{1}$ and $x_{1}^{*}=f_{1} \sin (2 \pi \vartheta)+$ $\varepsilon \xi_{1}^{*}$, where $\xi_{1}, \xi_{1}^{*}$ are independent standard Gaussian random variables. We define

$$
\bar{\vartheta}_{\varepsilon}=\frac{1}{2 \pi} \arctan \left(\frac{x_{1}^{*}}{x_{1}}\right)
$$

if $x_{1} \neq 0$ and $\bar{\vartheta}_{\varepsilon}=1 / 4$ if $x_{1}=0$. One easily checks that $\bar{\vartheta}_{\varepsilon}$ is the maximum likelihood estimator in the model induced by observations $\left(x_{1}, x_{1}^{*}\right)$. The following result describes its asymptotic behavior.

Proposition 3. If $\varepsilon$ is sufficiently small, then

$$
\sup _{|\vartheta| \leq T} \mathbf{P}_{\vartheta, f}\left(\left|\bar{\vartheta}_{\varepsilon}-\vartheta\right| \geq x\right) \leq \exp \left(-2(x / \varepsilon)^{2} f_{1}^{2} \cos ^{2}(2 \pi T)\right),
$$

for all $x \in[0,1 / 2]$ and for all $T<1 / 4$.

Proof. Let us introduce $X=\sqrt{\xi_{1}^{2}+\xi_{1}^{* 2}}$. One checks that $\sin \left[2 \pi\left(\bar{\vartheta}_{\varepsilon}-\vartheta\right)\right]=$ $\varepsilon\left(\xi_{1} \sin \left(2 \pi \bar{\vartheta}_{\varepsilon}\right)-\xi_{1}^{*} \cos \left(2 \pi \bar{\vartheta}_{\varepsilon}\right)\right) f_{1}^{-1}$. The Cauchy-Schwarz inequality implies that $\left|\sin \left[2 \pi\left(\bar{\vartheta}_{\varepsilon}-\vartheta\right)\right]\right| \leq \varepsilon X\left|f_{1}^{-1}\right|$. Since $\vartheta \in[-1 / 4,1 / 4]$ and $\bar{\vartheta}_{\varepsilon} \in[-T, T]$, we have $\frac{\sin \left[2 \pi\left(\bar{\vartheta}_{\varepsilon}-\vartheta\right)\right]}{2 \pi\left(\vartheta_{\varepsilon}-\vartheta\right)} \geq \cos (2 \pi T)$. Therefore,

$$
\begin{aligned}
\mathbf{P}_{\vartheta, f}\left(\left|\bar{\vartheta}_{\varepsilon}-\vartheta\right| \geq x\right) & \leq \mathbf{P}_{\vartheta, f}\left(\frac{\left|\sin \left[2 \pi\left(\bar{\vartheta}_{\varepsilon}-\vartheta\right)\right]\right|}{2 \pi \cos (2 \pi T)} \geq x\right) \\
& \leq \mathbf{P}_{\vartheta, f}\left(X \geq 2 x \varepsilon^{-1} \pi\left|f_{1}\right| \cos (2 \pi T)\right),
\end{aligned}
$$

and the fact that $X^{2} / 2$ follows the exponential law completes the proof. 
5.2. Proofs of Lemmas used in Proposition 1. Let us start with some basic facts that will be often used in the proofs. For any $n, m, p \in \mathbb{N}$, we have

$$
n^{p}(n-m) \geq \sum_{k=m+1}^{n} k^{p} \geq \frac{n^{p}(n-m)}{p+1} .
$$

Applying this inequality to $p=2$, we get

$$
\max _{k \in B_{j}}(2 \pi k)^{2} \leq 3 \sigma_{j}^{2} / T_{j} .
$$

Assume now that $\xi$ is a random variable of law $\mathcal{N}(0,1)$. For any $\sigma^{2} \leq 1 / 4$, we have $\left(1-2 \sigma^{2}\right)^{-1} \leq 2$ and $\left(1-2 \sigma^{2}\right)^{-1 / 2} \leq e^{2 \sigma^{2}}$, therefore

$$
\mathbf{E}\left[e^{(\mu+\sigma \xi)^{2}}\right]=\frac{e^{\frac{\mu^{2}}{\left(1-2 \sigma^{2}\right)}}}{\sqrt{1-2 \sigma^{2}}} \leq e^{\frac{\mu^{2}}{\left(1-2 \sigma^{2}\right)}+2 \sigma^{2}} \leq \exp \left(2 \mu^{2}+2 \sigma^{2}\right) .
$$

Using the more precise inequalities $\left(1-2 \sigma^{2}\right)^{-1} \leq 1+4 \sigma^{2}$ and $\log \left(1-2 \sigma^{2}\right)^{-1} \leq$ $2 \sigma^{2}+4 \sigma^{4}$, we get $\mathbf{E}\left[e^{(\mu+\sigma \xi)^{2}}\right] \leq \exp \left(\mu^{2}+\sigma^{2}+2 \sigma^{2}\left(2 \mu^{2}+\sigma^{2}\right)\right)$ or equivalently,

$$
\mathbf{E}\left[e^{2 \mu \sigma \xi+\sigma^{2}\left(\xi^{2}-1\right)}\right] \leq e^{2 \sigma^{2}\left(2 \mu^{2}+\sigma^{2}\right)} .
$$

Throughout this section, we assume that $\left\|f^{\left(\beta_{*}\right)}\right\|^{2}=\sum_{k}(2 \pi k)^{2 \beta_{*}} f_{k}^{2}<\infty$ for some $\beta_{*}>1, N_{\varepsilon}=\left[\left(\varepsilon^{2} \log \varepsilon^{-5}\right)^{-\frac{1}{2 \beta_{*}+1}}\right]$ and $h \in[0,1]^{N_{\varepsilon}}$ is a random vector depending on $\left(x_{k}, x_{k}^{*}\right)$ only via $x_{k}^{2}+x_{k}^{* 2}$. Without loss of generality, we give the proofs in the case $\vartheta=0$.

Lemma 1. Set $\hat{\beta}_{*}=\beta_{*} \wedge 1.5$. For all $\tau$ such that $4 \pi|\tau-\vartheta| \leq N_{\varepsilon}^{-1}$,

$$
\left|\Phi_{\varepsilon}^{\prime \prime}(\tau, h)+\sum_{k=1}^{N_{\varepsilon}}(2 \pi k)^{2} h_{k} f_{k}^{2}\right| \leq N_{\varepsilon}^{2-2 \hat{\beta}_{*}}\left(\left\|f^{\left(\beta_{*}\right)}\right\|+2 \pi \hat{X} / \sqrt{\log \varepsilon^{-5}}\right)^{2},
$$

where $\hat{X}=\max _{1 \leq k \leq N_{\varepsilon}} \sqrt{\xi_{k}^{2}+\xi_{k}^{* 2}}$.

Proof. One easily checks that

$$
\begin{aligned}
\Phi_{\varepsilon}^{\prime \prime}(\vartheta+\tau, h)= & -\sum_{k=1}^{N_{\varepsilon}}(2 \pi k)^{2} h_{k} f_{k}^{2} \cos [4 \pi k \tau] \\
& -2 \varepsilon \sum_{k=1}^{N_{\varepsilon}}(2 \pi k)^{2} h_{k} f_{k}\left(\xi_{k} \cos [4 \pi k \tau]+\xi_{k}^{*} \sin [4 \pi k \tau]\right) \\
& -\varepsilon^{2} \sum_{k=1}^{N_{\varepsilon}}(2 \pi k)^{2} h_{k}\left[\left(\xi_{k}^{2}-\xi_{k}^{* 2}\right) \cos [4 \pi k \tau]+2 \xi_{k}^{*} \xi_{k} \sin [4 \pi k \tau]\right] .
\end{aligned}
$$

On the one hand, thanks to inequality $|1-\cos x| \leq|x|$,

$$
\begin{aligned}
\left|\sum_{k=1}^{N_{\varepsilon}}(2 \pi k)^{2} h_{k} f_{k}^{2}(1-\cos [4 \pi k \tau])\right| & \leq 2 \tau \sum_{k=1}^{N_{\varepsilon}}(2 \pi k)^{3} f_{k}^{2} \\
& \leq 2 \tau\left(2 \pi N_{\varepsilon}\right)^{3-2 \hat{\beta}_{*}}\left\|f^{\left(\beta_{*}\right)}\right\|^{2} \\
& \leq N_{\varepsilon}^{2-2 \hat{\beta}_{*}}\left\|f^{\left(\beta_{*}\right)}\right\|^{2} .
\end{aligned}
$$


On the other hand, in view of the Cauchy-Schwarz inequality, it holds $\xi_{k} \cos [4 \pi k \tau]+$ $\xi_{k}^{*} \sin [4 \pi k \tau] \leq \hat{X}$ and $\left(\xi_{k}^{2}-\xi_{k}^{* 2}\right) \cos [4 \pi k \tau]+2 \xi_{k}^{*} \xi_{k} \sin [4 \pi k \tau] \leq \hat{X}^{2}$. Therefore, it holds

$$
\begin{array}{r}
\left|\sum_{k=1}^{N_{\varepsilon}}(2 \pi k)^{2} h_{k} f_{k}\left(\xi_{k} \cos [4 \pi k \tau]+\xi_{k}^{*} \sin [4 \pi k \tau]\right)\right| \\
\leq \hat{X} \sum_{k=1}^{N_{\varepsilon}}(2 \pi k)^{2}\left|f_{k}\right| \\
\leq \hat{X}\left\|f^{\left(\beta_{*}\right)}\right\| \sqrt{\sum_{k=1}^{N_{\varepsilon}}(2 \pi k)^{4-2 \beta_{*}}} \leq 2 \pi \hat{X} N_{\varepsilon}^{\frac{5}{2}-\hat{\beta}_{*}}\left\|f^{\left(\beta_{*}\right)}\right\|,
\end{array}
$$

and

$$
\left|\sum_{k=1}^{N_{\varepsilon}}(2 \pi k)^{2} h_{k}\left[\left(\xi_{k}^{2}-\xi_{k}^{* 2}\right) \cos [4 \pi k \tau]+2 \xi_{k}^{*} \xi_{k} \sin [4 \pi k \tau]\right]\right| \leq 4 \pi^{2} N_{\varepsilon}^{3} \hat{X}^{2} .
$$

Taking into account the identity $\varepsilon^{2} N_{\varepsilon}^{3}=N_{\varepsilon}^{2-2 \beta_{*}} / \log \left(\varepsilon^{-5}\right)$, for all $\tau$ verifying $|\tau| \leq$ $\left(4 \pi N_{\varepsilon}\right)^{-1}$, we get

$$
\left|\Phi_{\varepsilon}^{\prime \prime}(\vartheta+\tau, h)+\sum_{k=1}^{N_{\varepsilon}} h_{k}(2 \pi k)^{2} f_{k}^{2}\right| \leq N_{\varepsilon}^{2-2 \hat{\beta}_{*}}\left(\left\|f^{\left(\beta_{*}\right)}\right\|^{2}+2 \pi \hat{X} / \sqrt{\log \varepsilon^{-5}}\right)^{2}
$$

and the assertion of the lemma follows.

Lemma 2. Let $h \in[0,1]^{N_{\varepsilon}}$ be a random vector depending on $\left(x_{k}, x_{k}^{*}\right)$ only via $x_{k}^{2}+x_{k}^{* 2}$. For any $x \in\left[0, \sqrt{N_{\varepsilon} / 6}\right]$, it holds

$$
\mathbf{P}_{\vartheta, f}\left(\left|\Phi_{\varepsilon}^{\prime}(\vartheta, h)\right|>2 x \varepsilon\left(\left\|f^{\prime}\right\|+2 \pi \varepsilon N_{\varepsilon}^{3 / 2}\right)\right) \leq 2 e^{-x^{2}}
$$

Proof. The random variables $X_{k}=(2 \pi k)\left(f_{k}+\varepsilon \xi_{k}\right) \xi_{k}^{*}, k=1, \ldots, N_{\varepsilon}$ and $h_{1}, \ldots, h_{N}$ fulfill the conditions of Lemma 14 with $\varrho_{k}^{*}=1, T_{k}=1 /(2 \sqrt{2} \pi k \varepsilon), g_{k}^{2}=(2 \pi k)^{2}\left(f_{k}^{2}+\right.$ $\varepsilon^{2}$ ), since due to (17),

$$
\mathbf{E}\left[e^{t X_{k}}\right]=\mathbf{E}\left[e^{(2 \pi k t)^{2}\left(f_{k}+\varepsilon \xi_{k}\right)^{2} / 2}\right] \leq e^{(2 \pi k t)^{2}\left(f_{k}^{2}+\varepsilon^{2}\right)} .
$$

By definition, $\Phi_{\varepsilon}^{\prime}(\vartheta, h)=\varepsilon \sum_{k=1}^{N_{\varepsilon}} h_{k} X_{k}$, and therefore,

$$
\mathbf{P}_{\vartheta, f}\left(\left|\Phi_{\varepsilon}^{\prime}(\vartheta, h)\right| \geq 2 x \varepsilon\left(\sum_{k=1}^{N_{\varepsilon}}(2 \pi k)^{2}\left(f_{k}^{2}+\varepsilon^{2}\right)\right)^{1 / 2}\right) \leq 2 e^{-x^{2}}
$$

for all $x \in\left[0,\left(\sum_{k}(2 \pi k)^{2}\left(f_{k}^{2}+\varepsilon^{2}\right)\right)^{1 / 2} /\left(2 \sqrt{2} \pi N_{\varepsilon} \varepsilon\right)\right]$. To complete the proof, it suffices to remark that

$$
\frac{\sum_{k=1}^{N_{\varepsilon}}(2 \pi k)^{2}\left(f_{k}^{2}+\varepsilon^{2}\right)}{8 \pi^{2} N_{\varepsilon}^{2} \varepsilon^{2}} \geq \frac{\sum_{k=1}^{N_{\varepsilon}} k^{2}}{2 N_{\varepsilon}^{2}} \geq \frac{N_{\varepsilon}}{6}
$$

and $\sum_{k=1}^{N_{\varepsilon}}(2 \pi k)^{2}\left(f_{k}^{2}+\varepsilon^{2}\right) \leq\left(\left\|f^{\prime}\right\|+\varepsilon(2 \pi) N_{\varepsilon}^{3 / 2}\right)^{2}$.

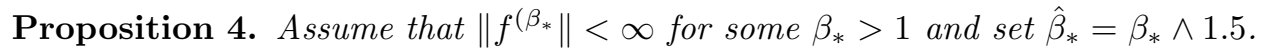
There exists an event $\mathcal{A}_{\varepsilon}$ such that for every $\varepsilon<1 / 2, \mathbf{P}_{\vartheta, f}\left(\mathcal{A}_{\varepsilon}\right) \geq 1-2 \varepsilon^{4}$ and on 
$\mathcal{A}_{\varepsilon}$ it holds:

$$
\begin{aligned}
\Phi_{\varepsilon}^{\prime \prime}(\tau, h) \leq & -\sum_{k=1}^{N_{\varepsilon}} h_{k}(2 \pi k)^{2} f_{k}^{2}+N_{\varepsilon}^{2-2 \hat{\beta}_{*}}\left(\left\|f^{\left(\beta_{*}\right)}\right\|+3 \pi\right)^{2} \\
\frac{\Phi_{\varepsilon}^{\prime}(\tau, h)}{\tau-\vartheta} \leq & -\sum_{k=1}^{N_{\varepsilon}} h_{k}(2 \pi k)^{2} f_{k}^{2}+N_{\varepsilon}^{2-2 \hat{\beta}_{*}}\left(\left\|f^{\left(\beta_{*}\right)}\right\|+3 \pi\right)^{2} \\
& +\frac{2 \varepsilon\left(\left\|f^{\prime}\right\| \sqrt{\log \varepsilon^{-5}}+2 \pi N_{\varepsilon}^{1-\beta_{*}}\right)}{|\tau-\vartheta|}
\end{aligned}
$$

for all $\tau \in\left[\vartheta-\left(4 \pi N_{\varepsilon}\right)^{-1}, \vartheta+\left(4 \pi N_{\varepsilon}\right)^{-1}\right]$.

Proof. According to Lemma 1, we have

$$
\Phi_{\varepsilon}^{\prime \prime}(\tau, h) \leq-\sum_{k=1}^{N_{\varepsilon}} h_{k}(2 \pi k)^{2} f_{k}^{2}+N_{\varepsilon}^{2-2 \hat{\beta}_{*}}\left(\left\|f^{\left(\beta_{*}\right)}\right\|+2 \pi \hat{X} / \sqrt{\log \varepsilon^{-5}}\right)^{2} .
$$

Since for every $k,\left(\xi_{k}^{2}+\xi_{k}^{* 2}\right) / 2$ follows the exponential law with mean 1 , we have

$$
\mathbf{P}\left(4 \hat{X}^{2}>9 \log \varepsilon^{-5}\right) \leq N_{\varepsilon} \mathbf{P}\left(X_{1}^{2} / 2>\log \varepsilon^{-5}\right) \leq N_{\varepsilon} \varepsilon^{5} \leq \varepsilon^{4} .
$$

This inequality completes the proof of (20).

To prove (21), note that for some $\tilde{\tau} \in[\vartheta, \tau]$, we have $\Phi_{\varepsilon}^{\prime}(\tau, h)=\Phi_{\varepsilon}^{\prime}(\vartheta, h)+(\tau-$ $\vartheta) \Phi_{\varepsilon}^{\prime \prime}(\tilde{\tau}, h)$. Lemma 2 and (20) yield (21).

5.3. Lemmas used in Theorem 1, Let us start with some simple algebra allowing to obtain a rough evaluation of $R^{\varepsilon}\left[f, h^{*}\right]$, where $h^{*}$ is the ideal filter an oracle would choose in the class of blockwise constant filters. For this filter $h^{*}$, it holds

$$
R^{\varepsilon}\left[f, h^{*}\right]=\sum_{j=1}^{J} \frac{\varepsilon^{2} \sigma_{j}^{2}\left\|f^{\prime}\right\|_{(j)}^{2}}{\left\|f^{\prime}\right\|_{(j)}^{2}+\varepsilon^{2} \sigma_{j}^{2}}+\sum_{k>N_{\varepsilon}}(2 \pi k)^{2} f_{k}^{2} .
$$

Using the explicit form of $h_{k}^{*}$, we get

$$
R^{\varepsilon}\left[f, h^{*}\right]=\sum_{k=1}^{\infty}\left(1-h_{k}^{*}\right)(2 \pi k)^{2} f_{k}^{2} \geq \varepsilon^{2} \sum_{k=1}^{N_{\varepsilon}} h_{k}^{*}(2 \pi k)^{2} .
$$

Since $\varepsilon^{2} N_{\varepsilon}^{3} \rightarrow 0$ as $\varepsilon \rightarrow 0$, we have $R^{\varepsilon}\left[f, h^{*}\right] \leq C N_{\varepsilon}^{2-2 \beta_{*}} \rightarrow 0$ as $\varepsilon \rightarrow 0$. In view of $\left\|f^{\prime}\right\|_{(1)}^{2} \geq 4 \pi^{2} \rho^{2}$ and $\varepsilon^{2} \sigma_{1}^{2} \leq 4 \pi^{2} \varepsilon^{2} N_{\varepsilon}^{3} \rightarrow 0$ as $\varepsilon \rightarrow 0$, for $\varepsilon$ small enough the inequality $\varepsilon^{2} \sigma_{1}^{2} \leq\left\|f^{\prime}\right\|_{(1)}^{2}$ holds. Therefore,

$$
R^{\varepsilon}\left[f, h^{*}\right] \geq \frac{\varepsilon^{2} \sigma_{1}^{2}\left\|f^{\prime}\right\|_{(1)}^{2}}{\left\|f^{\prime}\right\|_{(1)}^{2}+\varepsilon^{2} \sigma_{1}^{2}} \geq \frac{\varepsilon^{2} \sigma_{1}^{2}}{2} .
$$

Hence, for every function $f$, the quantity $R^{\varepsilon}\left[f, h^{*}\right]$ tends to zero as $\varepsilon \rightarrow 0$ slower than $\varepsilon^{2}$ and faster than $\varepsilon^{2} N_{\varepsilon}^{3}$.

Lemma 3. It holds $\mathbf{E}\left[\Phi_{\varepsilon}^{\prime}(\vartheta, \tilde{h})^{2}\right] \leq \varepsilon^{2} \sum_{k=1}^{N_{\varepsilon}} h_{k}^{* 2}(2 \pi k)^{2}\left(f_{k}^{2}+\varepsilon^{2}\right)$. 
Proof. Using (6), one checks that

$$
\Phi_{\varepsilon}^{\prime}(\vartheta, \tilde{h})=\varepsilon \sum_{k=1}^{N_{\varepsilon}} \tilde{h}_{k}(2 \pi k)\left(f_{k}+\varepsilon \xi_{k}(\vartheta)\right) \xi_{k}^{*}(\vartheta) .
$$

In the sequel, we write $\xi_{k}, \xi_{k}^{*}$ instead of $\xi_{k}(\vartheta), \xi_{k}^{*}(\vartheta)$. For any $k^{\prime} \neq k$, the random variable $\tilde{h}_{k}\left(f_{k}+\varepsilon \xi_{k}\right) \xi_{k}^{*} \tilde{h}_{k^{\prime}}\left(f_{k^{\prime}}+\varepsilon \xi_{k^{\prime}}\right) \xi_{k^{\prime}}^{*}$ is symmetric. Therefore it has zero mean and

$$
\begin{aligned}
\mathbf{E}\left[\Phi_{\varepsilon}^{\prime}(\vartheta, \tilde{h})^{2}\right] & =\varepsilon^{2} \sum_{k=1}^{N_{\varepsilon}}(2 \pi k)^{2} \mathbf{E}\left[\tilde{h}_{k}^{2}\left(f_{k}+\varepsilon \xi_{k}\right)^{2} \xi_{k}^{* 2}\right] \\
& \leq \varepsilon^{2} \sum_{k=1}^{N_{\varepsilon}}(2 \pi k)^{2} h_{k}^{* 2} \mathbf{E}\left[\left(f_{k}+\varepsilon \xi_{k}\right)^{2} \xi_{k}^{* 2}\right],
\end{aligned}
$$

and the assertion of the lemma follows.

Lemma 4. Let us denote

$$
\eta_{j}=\frac{2 \varepsilon \sum_{k \in B_{j}}(2 \pi k)^{2} f_{k} \xi_{k}+\varepsilon^{2} \sum_{k \in B_{j}}(2 \pi k)^{2}\left(\xi_{k}^{2}+\xi_{k}^{* 2}-2\right)}{\left\|f^{\prime}\right\|_{(j)}^{2}+\varepsilon^{2} \sigma_{j}^{2}} .
$$

For any positive $x$ such that $x^{2} \leq T_{j} / 10$, it holds

$$
\mathbf{P}\left(\left|\eta_{j}\right|>x \sqrt{24\left(1-h_{\kappa_{j}}^{*}\right) T_{j}^{-1}}\right) \leq 2 e^{-x^{2}}
$$

Proof. Set $Y_{k}=2 \varepsilon(2 \pi k)^{2} f_{k} \xi_{k}+\varepsilon^{2}(2 \pi k)^{2}\left(\xi_{k}^{2}+\xi_{k}^{* 2}-2\right), \sigma=\varepsilon(2 \pi k) \sqrt{t}$ and $\mu=$ $(2 \pi k) f_{k} \sqrt{t}$. Using (18) we infer that

$$
\begin{aligned}
\mathbf{E}\left[e^{t Y_{k}}\right] & =\mathbf{E}\left[e^{2 \mu \sigma \xi_{k}+\sigma^{2}\left(\xi_{k}^{2}-1\right)}\right] \mathbf{E}\left[e^{\sigma^{2}\left(\xi_{k}^{* 2}-1\right)}\right] \leq e^{4 \sigma^{2}\left(\mu^{2}+\sigma^{2}\right)} \\
& =e^{4 \varepsilon^{2}(2 \pi k)^{4} t^{2}\left(f_{k}^{2}+\varepsilon^{2}\right)}
\end{aligned}
$$

as soon as $\sigma=\varepsilon(2 \pi k) \sqrt{t} \leq 1 / 2$, or equivalently $t \leq 1 / 4 \varepsilon^{2}(2 \pi k)^{2}$. By [25, Thm. 2.7], for any $x>0$, we get

$$
\mathbf{P}\left(\left|\sum_{k \in B_{j}} Y_{j}\right| \geq x\left(2 \sum_{k \in B_{j}} 4 \varepsilon^{2}(2 \pi k)^{4}\left(f_{k}^{2}+\varepsilon^{2}\right)\right)^{1 / 2}\right) \leq 2 e^{-x^{2}\left(1 \wedge Q_{\varepsilon} x^{-1}\right)}
$$

where

$$
Q_{\varepsilon}=\frac{\left(8 \varepsilon^{2} \sum_{k \in B_{j}}(2 \pi k)^{4}\left(f_{k}^{2}+\varepsilon^{2}\right)\right)^{1 / 2}}{4\left(2 \varepsilon \pi \kappa_{j+1}\right)^{2}} .
$$

It is clear that

$$
\begin{gathered}
Q_{\varepsilon} \geq \frac{\left(8 \sum_{k \in B_{j}}(2 \pi k)^{4}\right)^{1 / 2}}{4\left(2 \pi \kappa_{j+1}\right)^{2}} \geq \frac{\left(8(2 \pi)^{4} \kappa_{j+1}^{4} T_{j} / 5\right)^{1 / 2}}{4\left(2 \pi \kappa_{j+1}\right)^{2}} \geq \sqrt{T_{j} / 10} \\
\sum_{k \in B_{j}}(2 \pi k)^{4}\left(f_{k}^{2}+\varepsilon^{2}\right) \leq\left(2 \pi \kappa_{j+1}\right)^{2}\left(\left\|f^{\prime}\right\|_{(j)}^{2}+\varepsilon^{2} \sigma_{j}^{2}\right) \leq 3 T_{j}^{-1} \sigma_{j}^{2}\left(\left\|f^{\prime}\right\|_{(j)}^{2}+\varepsilon^{2} \sigma_{j}^{2}\right) \\
\leq \frac{3\left(1-h_{\kappa_{j}}^{*}\right)\left(\left\|f^{\prime}\right\|_{(j)}^{2}+\varepsilon^{2} \sigma_{j}^{2}\right)^{2}}{T_{j} \varepsilon^{2}} .
\end{gathered}
$$

These inequalities combined with the identity $\eta_{j}=\sum_{k \in B_{j}} Y_{j} /\left(\left\|f^{\prime}\right\|_{(j)}^{2}+\varepsilon^{2} \sigma_{j}^{2}\right)$ yield the desired result. 
Lemma 5. Assume that $\varphi_{j}^{2} T_{j} \geq 24 \log \varepsilon^{-5}$ and $\varphi_{j}<1$. Then

$$
\mathbf{P}\left(\hat{h}_{k}^{J S} \in\left[h_{k}^{*}-\frac{2\left(1-h_{k}^{*}\right) \varphi_{j}}{\left(1-\varphi_{j}\right)}, h_{k}^{*}\right]\right) \geq 1-2 \varepsilon^{5} .
$$

Proof. Note that

$$
\hat{h}_{k}^{J S}=\left(1-\frac{\left(1-h_{k}^{*}\right)\left(1+\varphi_{j}\right)}{1+\eta_{j}}\right) \mathbb{1}_{\left\{\eta_{j}>-h_{k}^{*}+\varphi_{j}\left(1-h_{k}^{*}\right)\right\}} .
$$

One checks that $\hat{h}_{k}^{J S}>h_{k}^{*}$ if and only if $\eta_{j}>\varphi_{j}$. Therefore, $\mathbf{P}\left(\hat{h}_{k}^{J S}>h_{k}^{*}\right)=$ $\mathbf{P}\left(\eta_{j}>\varphi_{j}\right) \leq \varepsilon^{4}$. Similarly,

$$
\begin{aligned}
\mathbf{P}\left(\hat{h}_{k}^{J S}<h_{k}^{*}-\frac{2\left(1-h_{k}^{*}\right) \varphi_{j}}{\left(1-\varphi_{j}\right)}\right)= & \mathbf{P}\left(\varphi_{j}-\left(1+\varphi_{j}\right) h_{k}^{*} \leq \eta_{j} \leq-\varphi_{j}\right) \\
& +\mathbf{P}\left(\eta_{j}<\varphi_{j}-\left(1+\varphi_{j}\right) h_{k}^{*}\right) \mathbb{1}_{\left\{h_{k}^{*}>\frac{2\left(1-h_{k}^{*}\right) \varphi_{j}}{\left(1-\varphi_{j}\right)}\right\}} \\
\leq & \mathbf{P}\left(\eta_{j} \leq-\varphi_{j}\right)
\end{aligned}
$$

since $h_{k}^{*}>\frac{2\left(1-h_{k}^{*}\right) \varphi_{j}}{\left(1-\varphi_{j}\right)}$ if and only if $\varphi_{j}-\left(1+\varphi_{j}\right) h_{k}^{*}<-\varphi_{j}$. Therefore, using Lemma 4,

$$
\mathbf{P}\left(\hat{h}_{k}^{J S} \in\left[h_{k}^{*}-\frac{2\left(1-h_{k}^{*}\right) \varphi_{j}}{\left(1-\varphi_{j}\right)}, h_{k}^{*}\right]\right) \geq \mathbf{P}\left(\left|\eta_{j}\right| \leq \varphi_{j}\right) \geq 1-2 \varepsilon^{5}
$$

and the assertion of the lemma follows.

Lemma 6. For any positive $x$ verifying $x^{2} \leq T_{\varepsilon} / 5$ it holds

$$
\mathbf{P}\left(\left|\sum_{k=1}^{N_{\varepsilon}} \tilde{h}_{k}(2 \pi k)^{2}\left(\xi_{k}^{2}-\xi_{k}^{* 2}\right)\right| \geq \frac{12 \sqrt{2} x}{\sqrt{T_{\varepsilon}}} R^{\varepsilon}\left[f, h^{*}\right]\right) \leq 2 J e^{-x^{2}} .
$$

Proof. Using (16), we get $\left(\sum_{k \in B_{j}}(2 \pi k)^{4}\right)^{1 / 2} \leq 3 \sigma_{j}^{2} / \sqrt{T_{j}}$. This inequality combined with the fact that $\tilde{h}_{k} \leq h_{k}^{*}$, allows us to bound the probability of the event of interest by

$$
\begin{gathered}
\mathbf{P}\left(\left|\sum_{k=1}^{N_{\varepsilon}} \tilde{h}_{k}(2 \pi k)^{2}\left(\xi_{k}^{2}-\xi_{k}^{* 2}\right)\right| \geq \sqrt{32} x \sum_{j=1}^{J} h_{\kappa_{j}}^{*}\left(\sum_{k \in B_{j}}(2 \pi k)^{4}\right)^{\frac{1}{2}}\right) \\
\leq \sum_{j=1}^{J} \mathbf{P}\left(\tilde{h}_{\kappa_{j}}\left|\sum_{k \in B_{j}}(2 \pi k)^{2}\left(\xi_{k}^{2}-\xi_{k}^{* 2}\right)\right| \geq \sqrt{32} x h_{\kappa_{j}}^{*}\left(\sum_{k \in B_{j}}(2 \pi k)^{4}\right)^{1 / 2}\right) \\
\leq \sum_{j=1}^{J} \mathbf{P}\left(\left|\sum_{k \in B_{j}}(2 \pi k)^{2}\left(\xi_{k}^{2}-\xi_{k}^{* 2}\right)\right| \geq \sqrt{32} x\left(\sum_{k \in B_{j}}(2 \pi k)^{4}\right)^{1 / 2}\right) .
\end{gathered}
$$

The desired result follows now from Lemma 13 and (15).

Lemma 7. For any $x>0$,

$$
\mathbf{P}\left(\varepsilon\left|\sum_{k=1}^{N_{\varepsilon}}\left(\tilde{h}_{k}-h_{k}^{*}\right)(2 \pi k)^{2} \xi_{k} f_{k}\right| \geq 5 x \sqrt{\frac{\varphi_{\varepsilon}}{T_{\varepsilon}}} R^{\varepsilon}\left[f, h^{*}\right]\right) \leq 2 J e^{-x^{2}}
$$


Proof. Remark first that

$$
\left(\tilde{h}_{\kappa_{j}}-h_{\kappa_{j}}^{*}\right)^{2} \leq h_{\kappa_{j}}^{* 2} \wedge 8\left(1-h_{\kappa_{j}}^{*}\right)^{2} \varphi_{j}^{2} \leq \sqrt{8} \varphi_{j} h_{\kappa_{j}}^{*}\left(1-h_{\kappa_{j}}^{*}\right)
$$

for all $j=1, \ldots, J$. Set $Y_{j}=\varepsilon \sum_{k \in B_{j}}(2 \pi k)^{2} \xi_{k} f_{k}$. The random variables $Y_{1}, \ldots, Y_{J}$ are independent zero mean Gaussian with variance

$$
\mathbf{E}\left[Y_{j}^{2}\right]=\varepsilon^{2} \sum_{k \in B_{j}}(2 \pi k)^{4} f_{k}^{2} \leq 3 \varepsilon^{2} \sigma_{j}^{2} T_{j}^{-1} \sum_{k \in B_{j}}(2 \pi k)^{2} f_{k}^{2} .
$$

Therefore, $\mathbf{P}\left(\left|Y_{j}\right| \geq \sqrt{6 / T_{j}} x \varepsilon \sigma_{j}\left\|f^{\prime}\right\|_{(j)}\right) \leq 2 e^{-x^{2}}$ and consequently,

$$
\mathbf{P}\left(\sum_{j=1}^{n}\left|\left(\tilde{h}_{\kappa_{j}}-h_{\kappa_{j}}^{*}\right) Y_{j}\right| \geq \sqrt{\frac{6}{T_{\varepsilon}}} x \varepsilon \sum_{j=1}^{J} \sqrt{4 \varphi_{j} h_{\kappa_{j}}^{*}\left(1-h_{\kappa_{j}}^{*}\right)} \sigma_{j}\left\|f^{\prime}\right\|_{(j)}\right) \leq 2 J e^{-x^{2}} .
$$

To complete the proof, note that

$$
\varepsilon \sum_{j=1}^{J} \sqrt{h_{\kappa_{j}}^{*}\left(1-h_{\kappa_{j}}^{*}\right)} \sigma_{j}\left\|f^{\prime}\right\|_{(j)} \leq \frac{1}{2} \sum_{j=1}^{J}\left(1-h_{\kappa_{j}}^{*}\right)\left\|f^{\prime}\right\|_{(j)}^{2}+\frac{1}{2} \sum_{j=1}^{J} h_{\kappa_{j}}^{*}\left(\varepsilon \sigma_{j}\right)^{2}
$$

and the right side is bounded by $R^{\varepsilon}\left[f, h^{*}\right]$.

Lemma 8. We have $\sum_{k=1}^{N_{\varepsilon}}\left(\tilde{h}_{k}-h_{k}^{*}\right)(2 \pi k)^{2} f_{k}^{2} \leq 4 \varphi_{\varepsilon} R^{\varepsilon}\left[f, h^{*}\right]$.

Proof. The desired inequality is trivially fulfilled on $\mathcal{E}^{c}$, while on $\mathcal{E}$ we have $0 \leq$ $h_{k}^{*}-\tilde{h}_{k} \leq 4 \varphi_{\varepsilon}\left(1-h_{k}^{*}\right), \forall k \in B_{j}$, and hence $\quad \sum_{k=1}^{\infty}\left|\tilde{h}_{k}-h_{k}^{*}\right|(2 \pi k)^{2} f_{k}^{2} \leq 4 \varphi_{\varepsilon} \sum_{k=1}^{N_{\varepsilon}}(1-$ $\left.h_{k}^{*}\right)(2 \pi k)^{2} f_{k}^{2} \leq 4 \varphi_{\varepsilon} R^{\varepsilon}\left[f, h^{*}\right]$.

Lemma 9. Set $\hat{X}=\max _{1 \leq k \leq N_{\varepsilon}} \sqrt{\xi_{k}^{2}(\vartheta)+\xi_{k}^{* 2}(\vartheta)}$. There exists an event of probability at least $1-2 \varepsilon^{4}$ such that on this event, for all $\tau \in \mathbb{R}$, we have

$$
\begin{aligned}
\frac{\left|\Phi_{\varepsilon}^{\prime \prime \prime}(\tau, \tilde{h})\right|}{R^{\varepsilon}\left[f, h^{*}\right]} \leq & \frac{12|\tau-\vartheta| \cdot\left\|f^{\prime}\right\|^{2}}{\varepsilon^{2} T_{\varepsilon}}+\frac{12\left\|f^{\prime}\right\| \sqrt{\log \varepsilon^{-8}}}{\varepsilon T_{\varepsilon}} \\
& +\frac{16 \sqrt{3} \pi N_{\varepsilon}|\tau-\vartheta| \hat{X}\left\|f^{\prime}\right\|}{\varepsilon \sqrt{T_{\varepsilon}}}+4 \pi N_{\varepsilon} \hat{X}^{2} .
\end{aligned}
$$

Proof. Using (6), one checks that

$$
\begin{aligned}
\Phi_{\varepsilon}^{\prime \prime \prime}(\tau, h)= & 2 \sum_{k=1}^{\infty} h_{k}(2 \pi k)^{3}\left[\left(f_{k}+\varepsilon \xi_{k}(\vartheta)\right)^{2}-\varepsilon^{2} \xi_{k}^{*}(\vartheta)^{2}\right] \sin [4 \pi k(\tau-\vartheta)] \\
& -4 \varepsilon \sum_{k=1}^{\infty} h_{k}(2 \pi k)^{3}\left(f_{k}+\varepsilon \xi_{k}(\vartheta)\right) \xi_{k}^{*}(\vartheta) \cos [4 \pi k(\tau-\vartheta)] .
\end{aligned}
$$


Without loss of generality, we assume in the sequel that $\vartheta=0$. Then

$$
\begin{aligned}
\left|\Phi_{\varepsilon}^{\prime \prime \prime}(\tau, \tilde{h})\right| \leq 2 & \sum_{k=1}^{N_{\varepsilon}} \tilde{h}_{k}(2 \pi k)^{3} f_{k}^{2}|\sin (4 \pi k \tau)|+4 \varepsilon \sum_{k=1}^{N_{\varepsilon}} \tilde{h}_{k}(2 \pi k)^{3}\left|f_{k} \xi_{k}^{*}\right| \\
& +4 \varepsilon \sum_{k=1}^{N_{\varepsilon}} \tilde{h}_{k}(2 \pi k)^{3}\left|f_{k}\left[\xi_{k} \sin (4 \pi k \tau)+\xi_{k}^{*}(\cos (4 \pi k \tau)-1)\right]\right| \\
& +2 \varepsilon^{2} \sum_{k=1}^{N_{\varepsilon}} \tilde{h}_{k}(2 \pi k)^{3}\left|\left(\xi_{k}^{2}-\xi_{k}^{* 2}\right) \sin (4 \pi k \tau)-2 \xi_{k} \xi_{k}^{*} \cos (4 \pi k \tau)\right| .
\end{aligned}
$$

Using the inequalities $|\sin (4 \pi k \tau)| \leq 4 \pi k|\tau|$ and

$$
\sum_{k=1}^{N_{\varepsilon}} \tilde{h}_{k}(2 \pi k)^{4} f_{k}^{2} \leq \sum_{j=1}^{J} h_{\kappa_{j}}^{*}\left\|f^{\prime}\right\|_{(j)}^{2}\left(2 \pi \kappa_{j+1}\right)^{2} \leq \frac{3\left\|f^{\prime}\right\|^{2}}{T_{\varepsilon}} \sum_{j=1}^{J} h_{\kappa_{j}}^{*} \sigma_{j}^{2},
$$

as well as the inequality $\varepsilon^{2} \sum_{j=1}^{J} h_{\kappa_{j}}^{*} \sigma_{j}^{2} \leq R^{\varepsilon}\left[f, h^{*}\right]$, we get the desired bound for the first sum. The bound on the second term is obtained using Lemma 14, the well known bound on the Laplace transform of a Gaussian distribution and the inequality

$$
\begin{aligned}
\sum_{k=1}^{N_{\varepsilon}} h_{k}^{* 2}(2 \pi k)^{6} f_{k}^{2} & \leq \sum_{j=1}^{J} h_{\kappa_{j}}^{* 2}\left(2 \pi \kappa_{j+1}\right)^{4}\left\|f^{\prime}\right\|_{(j)}^{2} \leq \frac{9}{T_{\varepsilon}^{2}} \sum_{j=1}^{J} h_{\kappa_{j}}^{* 2} \sigma_{j}^{4}\left\|f^{\prime}\right\|_{(j)}^{2} \\
& \leq \frac{9\left\|f^{\prime}\right\|^{2}}{T_{\varepsilon}^{2}} \max _{j} h_{\kappa_{j}}^{* 2} \sigma_{j}^{4} \leq \frac{9\left\|f^{\prime}\right\|^{2} R^{\varepsilon}\left[f, h^{*}\right]^{2}}{\varepsilon^{4} T_{\varepsilon}^{2}} .
\end{aligned}
$$

The bounds on the two remaining sums are obtained by combining the inequalities

$$
\begin{aligned}
&\left|\xi_{k} \sin (4 \pi k \tau)+\xi_{k}^{*}(\cos (4 \pi k \tau)-1)\right| \leq|4 \pi k \tau| \cdot\left|\xi_{k} \cos (4 \pi k \tilde{\tau})-\xi_{k}^{*} \sin (4 \pi k \tilde{\tau})\right| \\
& \leq 4 \pi k|\tau| \hat{X}, \\
&\left|\left(\xi_{k}^{2}-\xi_{k}^{* 2}\right) \sin (4 \pi k \tau)-2 \xi_{k} \xi_{k}^{*} \cos (4 \pi k \tau)\right| \leq \hat{X}^{2}
\end{aligned}
$$

with arguments similar to those used to bound the first two sums.

Lemma 10. For any $x>0$, it holds

$$
\mathbf{P}\left(\left|\Phi_{\varepsilon}^{\prime}(\vartheta, \tilde{h})-\Phi_{\varepsilon}^{\prime}\left(\vartheta, h^{*}\right)\right|^{2} \geq 12 \varepsilon^{2} x^{2} \varphi_{\varepsilon} R^{\varepsilon}\left[f, h^{*}\right]\right) \leq 2 e^{-x^{2}\left(1 \wedge \sqrt{T_{\varepsilon} / 8 x^{2}}\right)} .
$$

Proof. Let us denote $X_{k}=(2 \pi k)\left(f_{k}+\varepsilon \xi_{k}\right) \xi_{k}^{*}$. According to (24),

$$
\Phi_{\varepsilon}^{\prime}(\vartheta, \tilde{h})-\Phi_{\varepsilon}^{\prime}\left(\vartheta, h^{*}\right)=\varepsilon \sum_{j=1}^{k}\left(\tilde{h}_{k}-h_{k}^{*}\right) X_{k} .
$$

According to (19), for all $t \leq 1 /(2 \sqrt{2} \pi k \varepsilon)$, we have $\mathbf{E}\left[e^{t X_{k}}\right] \leq e^{t^{2}(2 \pi k)^{2}\left(f_{k}^{2}+\varepsilon^{2}\right)}$. Thus the conditions of Lemma 14 are fulfilled with $\varrho_{k}=\tilde{h}_{k}-h_{k}^{*}, \varrho_{k}^{*}=3 \sqrt{h_{k}^{*} \varphi_{j}\left(1-h_{k}^{*}\right)}$, $T_{k}=1 /(\sqrt{8} \pi k \varepsilon)$ and $g_{k}^{2}=(2 \pi k)^{2}\left(f_{k}^{2}+\varepsilon^{2}\right)$. Therefore,

$$
\mathbf{P}\left(\left|\Phi_{\varepsilon}^{\prime}(\vartheta, \tilde{h})-\Phi_{\varepsilon}^{\prime}\left(\vartheta, h^{*}\right)\right|^{2} \geq 12 \varepsilon^{2} x^{2} \varphi_{\varepsilon} \sum_{k=1}^{N_{\varepsilon}}(2 \pi k)^{2} h_{k}^{*}\left(1-h_{k}^{*}\right)\left(f_{k}^{2}+\varepsilon^{2}\right)\right) \leq 2 e^{-x^{2}}
$$


for any $x>0$ verifying

$$
x^{2} \leq \frac{3 \sum_{k=1}^{N_{\varepsilon}} \varphi_{j(k)}(2 \pi k)^{2} h_{k}^{*}\left(1-h_{k}^{*}\right)\left(f_{k}^{2}+\varepsilon^{2}\right)}{8 \varepsilon^{2} \max _{j} \varphi_{j} h_{\kappa_{j}}^{*}\left(1-h_{\kappa_{j}}^{*}\right)\left(2 \pi \kappa_{j+1}\right)^{2}} .
$$

To complete the proof, it suffices to remark that

$$
\sum_{k=1}^{N_{\varepsilon}}(2 \pi k)^{2} h_{k}^{*}\left(1-h_{k}^{*}\right)\left(f_{k}^{2}+\varepsilon^{2}\right)=\sum_{j=1}^{J} h_{\kappa_{j}}^{*}\left(1-h_{\kappa_{j}}^{*}\right)\left[\left\|f^{\prime}\right\|_{(j)}^{2}+\varepsilon^{2} \sigma_{j}^{2}\right] \leq R^{\varepsilon}\left[f, h^{*}\right]
$$

and

$$
\frac{3 \sum_{k=1}^{N_{\varepsilon}} \varphi_{j(k)}(2 \pi k)^{2} h_{k}^{*}\left(1-h_{k}^{*}\right)\left(f_{k}^{2}+\varepsilon^{2}\right)}{8 \varepsilon^{2} \max _{j} \varphi_{j} h_{\kappa_{j}}^{*}\left(1-h_{\kappa_{j}}^{*}\right)\left(2 \pi \kappa_{j+1}\right)^{2}} \geq \frac{3 \max _{j} \varphi_{j} h_{\kappa_{j}}^{*}\left(1-h_{\kappa_{j}}^{*}\right) \sigma_{j}^{2}}{8 \max _{j} \varphi_{j} h_{\kappa_{j}}^{*}\left(1-h_{\kappa_{j}}^{*}\right)\left(2 \pi \kappa_{j+1}\right)^{2}} .
$$

Since $\sigma_{j}^{2} \geq T_{j}\left(2 \pi \kappa_{j+1}\right)^{2} / 3$, the assertion of the lemma follows.

Lemma 11. Let $\zeta=2 \varepsilon\left\|f^{\prime}\right\|^{-2} \sum_{k} h_{k}^{*}(2 \pi k)^{2} f_{k} \xi_{k}$. For any event $\mathcal{A}$ verifying $\mathbf{P}\left(\mathcal{A}^{c}\right)=O\left(\varepsilon^{4}\right)$, we have

$$
\mathbf{E}\left[\Phi_{\varepsilon}^{\prime}\left(\vartheta, h^{*}\right)^{2} \zeta \mathbb{1}_{\mathcal{A}}\right]=o\left(\varepsilon^{2} R^{\varepsilon}\left[f, h^{*}\right]\right) .
$$

Proof. We have

$$
\begin{aligned}
\mathbf{E}\left[\Phi_{\varepsilon}^{\prime}\left(\vartheta, h^{*}\right)^{2} \zeta\right] & =\varepsilon^{2} \mathbf{E}\left[\sum_{k=1}^{N_{\varepsilon}}(2 \pi k)^{2} h_{k}^{* 2}\left(f_{k}+\varepsilon \xi_{k}(\vartheta)\right)^{2} \xi_{k}^{*}(\vartheta)^{2} \zeta\right] \\
& =2 \varepsilon^{3} \mathbf{E}\left[\sum_{k=1}^{N_{\varepsilon}}(2 \pi k)^{2} h_{k}^{* 2} f_{k} \xi_{k} \zeta\right]=4 \varepsilon^{4}\left\|f^{\prime}\right\|^{-2} \sum_{k=1}^{N_{\varepsilon}}(2 \pi k)^{4} h_{k}^{* 3} f_{k}^{2} \\
& \leq 12 T_{\varepsilon}^{-1} \varepsilon^{4} \sum_{j=1}^{J} h_{\kappa_{j}}^{*} \sigma_{j}^{2} \leq 12 T_{\varepsilon}^{-1} \varepsilon^{2} R^{\varepsilon}\left[f, h^{*}\right] .
\end{aligned}
$$

Using the Rosenthal inequality, one easily checks that $\mathbf{E}\left[\Phi_{\varepsilon}^{\prime}\left(\vartheta, h^{*}\right)^{2 p}\right]=O\left(\varepsilon^{2 p}\right)$ and $\mathbf{E}\left[\zeta^{2 p}\right]=o\left(R^{\varepsilon}\left[f, h^{*}\right]^{p}\right)$ for any integer $p>0$. Therefore, the Cauchy-Schwarz inequality yields,

$$
\left|\mathbf{E}\left[\Phi_{\varepsilon}^{\prime}\left(\vartheta, h^{*}\right)^{2} \zeta \mathbb{1}_{\mathcal{A}^{c}}\right]\right| \leq o\left(\varepsilon^{2} \sqrt{R^{\varepsilon}\left[f, h^{*}\right]}\right) \sqrt{\mathbf{P}\left(\mathcal{A}^{c}\right)}=o\left(\varepsilon^{4} \sqrt{R^{\varepsilon}\left[f, h^{*}\right]}\right)
$$

and the assertion of the lemma follows.

5.4. Lemma used in Theorem 2. We assume that $f \in \mathcal{F}_{\delta, \beta, L}(\bar{f})$ with $\bar{f} \in$ $\mathcal{F}\left(\beta^{*}, L^{*}, \rho\right)$ and $\beta^{*}>\beta \geq \beta_{*}$. For the sake of completeness we give below a suitable version of the Pinsker theorem [27.

Lemma 12. Set $\gamma_{\varepsilon}=1 / \log \varepsilon^{-2}, W_{\varepsilon}=\left(\frac{L}{\varepsilon^{2}} \frac{(\beta+2)(2 \beta+1)}{(2 \pi)^{2 \beta}(\beta-1)}\right)^{1 /(2 \beta+1)}$ and define

$$
\lambda_{k}^{*}= \begin{cases}1, & k \leq \gamma_{\varepsilon} W_{\varepsilon}, \\ {\left[1-\left(\frac{k}{W_{\varepsilon}}\right)^{\beta-1}\right]_{+},} & k>\gamma_{\varepsilon} W_{\varepsilon} .\end{cases}
$$

The filter $\lambda_{k}^{*}$ satisfies

$$
\sup _{f \in \mathcal{F}_{\delta, \beta, L}} R^{\varepsilon}\left[f, \lambda^{*}\right] \leq(1+o(1)) C(\beta, L) \varepsilon^{\frac{4 \beta-4}{2 \beta+1}} .
$$


Proof. Set $v=f-\bar{f}$. Using the inequality $\left(\bar{f}_{k}+v_{k}\right)^{2} \leq 2 z^{-1} \bar{f}_{k}^{2}+(1+z) v_{k}^{2}$, $\forall z \in[0,1]$, we obtain

$$
\begin{aligned}
R^{\varepsilon}\left[f, \lambda^{*}\right] & =\sum_{k>\gamma_{\varepsilon} W_{\varepsilon}}(2 \pi k)^{2}\left(1-\lambda_{k}^{*}\right)^{2}\left(\bar{f}_{k}+v_{k}\right)^{2}+\varepsilon^{2} \sum_{k=1}^{\infty}(2 \pi k)^{2} \lambda_{k}^{* 2} \\
& \leq 2 z^{-1} \sum_{k>\gamma_{\varepsilon} W_{\varepsilon}}(2 \pi k)^{2}\left(1-\lambda_{k}^{*}\right)^{2} \bar{f}_{k}^{2}+(1+z) R^{\varepsilon}\left[v, \lambda^{*}\right] .
\end{aligned}
$$

Since $\bar{f} \in \mathcal{F}\left(\beta^{*}, L^{*}, \rho\right)$, we have

$$
\sum_{k>\gamma_{\varepsilon} W_{\varepsilon}}(2 \pi k)^{2}\left(1-\lambda_{k}^{*}\right)^{2} \bar{f}_{k}^{2} \leq L^{*}\left(\gamma_{\varepsilon} W_{\varepsilon}\right)^{2-2 \beta^{*}}=o\left(W_{\varepsilon}^{2-2 \beta}\right) .
$$

On the other hand, setting $\tilde{\lambda}_{k}^{*}=\left(1-\left(k / W_{\varepsilon}\right)^{\beta-1}\right)_{+}$,

$$
R^{\varepsilon}\left[v, \lambda^{*}\right]-R^{\varepsilon}\left[v, \tilde{\lambda}^{*}\right] \leq \varepsilon^{2} \sum_{k \leq \gamma_{\varepsilon} W_{\varepsilon}}(2 \pi k)^{2} \leq 4 \pi^{2} \varepsilon^{2}\left(\gamma_{\varepsilon} W_{\varepsilon}\right)^{3}=o\left(\varepsilon^{2} W_{\varepsilon}^{3}\right) .
$$

Using the relation $W_{\varepsilon}^{2-2 \beta}=O\left(\varepsilon^{2} W_{\varepsilon}^{3}\right)$ and choosing $z=z_{\varepsilon}$ appropriately, we get

$$
\sup _{f \in \mathcal{F}_{\delta, \beta, L}(\bar{f})} R^{\varepsilon}\left[f, \lambda^{*}\right] \leq(1+o(1)) \sup _{v \in \mathcal{W}(\beta, L)} R^{\varepsilon}\left[v, \tilde{\lambda}^{*}\right]+o\left(\varepsilon^{2} W_{\varepsilon}^{3}\right),
$$

where $\mathcal{W}(\beta, L)$ is the Sobolev ball $\left\{v: \sum_{k>1}(2 \pi k)^{2 \beta} f_{k}^{2} \leq L\right\}$. It then follows from [1, Thm. 1 and Example 1, p. 265] (with $\alpha=\beta-1$ and $\delta=3$ ) that $\sup _{v \in \mathcal{W}(\beta, L)} R^{\varepsilon}\left[v, \tilde{\lambda}^{*}\right]=C(\beta, L) \varepsilon^{\frac{4 \beta-4}{2 \beta+1}}(1+o(1))$. To conclude, it suffices to remark that $o\left(\varepsilon^{2} W_{\varepsilon}^{3}\right)=o\left(\varepsilon^{\frac{4 \beta-4}{2 \beta+1}}\right)$.

\subsection{Auxiliary general results.}

Lemma 13. Assume that $a_{1}, a_{2}, \ldots, a_{n} \in \mathbb{R}$ and $\varsigma=\sum_{k=1}^{n} a_{k}\left(\xi_{k}^{2}-\xi_{k}^{* 2}\right)$, where $\left(\xi_{1}, \ldots, \xi_{n}, \xi_{1}^{*}, \ldots, \xi_{n}^{*}\right)$ is a zero mean Gaussian vector with identity covariance matrix. For any $y \in\left[0,\|a\| / \max _{k} a_{k}\right]$, it holds

$$
\mathbf{P}\left(\varsigma^{2} \geq 32 y^{2} \sum_{k=1}^{n} a_{k}^{2}\right) \leq 2 e^{-y^{2}} .
$$

Proof. Using the formula of the Laplace transform of a chi-squared distribution, for any $t \in\left[-\left(\sqrt{8} a_{k}\right)^{-1},\left(\sqrt{8} a_{k}\right)^{-1}\right]$, we get

$$
\mathbf{E}\left[e^{a_{k} t\left(\xi_{k}^{2}-\xi_{k}^{* 2}\right)}\right]=\frac{1}{1-4 a_{k}^{2} t^{2}} \leq e^{8 a_{k}^{2} t^{2}} .
$$

Applying [25, Thm. 2.7] with $g_{k}=16 a_{k}^{2}$ and $x=\sqrt{32} y\|a\|$, we get the desired result.

Lemma 14. Let $X_{1}, \ldots, X_{n}$ be independent symmetric random variables. Let $\varrho=\left(\varrho_{1}, \ldots, \varrho_{n}\right)$ be a random vector satisfying $\left|\varrho_{j}\right| \leq \varrho_{j}^{*}, \forall j=1, \ldots, n$ with some deterministic sequence $\left(\varrho_{j}^{*}\right)_{j=1}^{n}$ and $\mathscr{L}\left(\varrho \mid X_{j}=x\right)=\mathscr{L}\left(\varrho \mid X_{j}=-x\right)$ for all $j \in\{1, \ldots, n\}$. If

$$
\mathbf{E}\left[e^{t X_{j}}\right] \leq e^{t^{2} g_{j}^{2}}
$$


for some sequence $\left(g_{j}\right)_{j=1, \ldots, n}$ and for $|t| \leq T_{j}$, then

$$
\mathbf{P}\left(\left|\sum_{j=1}^{n} \varrho_{j} X_{j}\right|^{2} \geq 4 x^{2} \sum_{j=1}^{n} \varrho_{j}^{* 2} g_{j}^{2}\right) \leq 2 e^{-x^{2}\left(1 \wedge Q_{n} x^{-1}\right)}, \forall x>0
$$

where $Q_{n}=\left(\sum_{j=1}^{n} \varrho_{j}^{* 2} g_{j}^{2}\right)^{1 / 2} \min _{j}\left(T_{j} / \varrho_{j}^{*}\right)$.

Proof. Set $Y_{j}=\varrho_{j} X_{j}$ and $\bar{Y}_{j}=\varrho_{j}^{*} X_{j}$. For any $p_{1}, \ldots, p_{n} \in \mathbb{N}^{n}$, the expectation $\mathbf{E}\left[Y_{1}^{p_{1}} \cdot \ldots \cdot Y_{n}^{p_{n}}\right]$ vanishes if at least one $p_{j}$ is odd. Therefore, $\mathbf{E}\left[\left(\sum_{j} Y_{j}\right)^{k}\right]=0$ if $k$ is odd and $\mathbf{E}\left[\left(\sum_{j} Y_{j}\right)^{k}\right] \leq \mathbf{E}\left[\left(\sum_{j} \bar{Y}_{j}\right)^{k}\right]$ if $k$ is even. Hence

$$
\begin{aligned}
\mathbf{E}\left[\exp \left(t \sum_{j} Y_{j}\right)\right] & =\sum_{k=0}^{\infty} \frac{t^{k} \mathbf{E}\left[\left(\sum_{j} Y_{j}\right)^{k}\right]}{k !} \leq \sum_{k=0}^{\infty} \frac{t^{2 k} \mathbf{E}\left[\left(\sum_{j} \bar{Y}_{j}\right)^{2 k}\right]}{(2 k) !} \\
& =\mathbf{E}\left[e^{t \sum_{j} \varrho_{j}^{*} X_{j}}\right] \leq e^{t^{2} \sum_{j} \varrho_{j}^{* 2} g_{j}^{2}}, \quad \forall|t| \leq \min _{j}\left(T_{j} / \varrho_{j}^{*}\right) .
\end{aligned}
$$

According to the Markov inequality, for every $t>0$,

$$
\mathbf{P}\left(\left|\sum_{j=1}^{n} \varrho_{j} X_{j}\right| \geq y\right) \leq 2 e^{-t y} \mathbf{E}\left[e^{t \sum_{j} Y_{j}}\right] \leq 2 \exp \left(-t y+t^{2} \sum_{j=1}^{n} \varrho_{j}^{* 2} g_{j}^{2}\right) .
$$

Setting $t=\left(x \wedge Q_{n}\right) /\left(\sum_{j} \varrho_{j}^{* 2} g_{j}^{2}\right)^{1 / 2}$ and $y^{2}=4 x^{2} \sum_{j} \varrho_{j}^{* 2} g_{j}^{2}$ we get the desired result.

Aknowledgement. We are thankful to the anonymous referee for the remarks that helped to improve the presentation.

\section{REFERENCES}

[1] Belitser, E. And Levit, B. (1995). On minimax filtering on ellipsoids. Math. Methods Statist. 4, 259-273.

[2] Bickel, P. (1982). On adaptive estimation. Ann. Statist. 10, 647-671.

[3] Bickel, P., Klahssen, C., Ritov, Y. and Wellner, J. (1998). Efficient and Adaptive Estimation for Semiparametric Models, Springer, New York.

[4] Brown, L., Low, M. And ZhaO, L. (1997). Superefficiency in nonparametric function estimation. Ann. Statist. 25, 2607-2625.

[5] CAI, T. (1999). Adaptive wavelet estimation: a block thresholding and oracle inequality approach. Ann. Statist. 27, 898-924.

[6] CaI, T. AND Low, M. (2005). Nonparametric estimation over shrinking neighborhoods: superefficiency and adaptation. Ann. Statist. 33 (1), 184-213.

[7] Castillo, I. (2005) Semiparametric second order efficient estimation of the period of a signal. To appear in Bernoulli.

[8] Cavalier, L. And Tsybakov, A. (2001). Penalized blockwise Stein's method, monotone oracles and sharp adaptive estimation. Math. Methods Statist. 10, 247-282.

[9] Cavalier, L. And Tsybakov, A. (2002). Sharp adaptation for inverse problems with random noise. Proba. Theory and Related Fields 123, 323-354.

[10] Dalalyan, A., Golubev, G. and Tsybakov, A. (2006). Penalized Maximum Likelihood and Semiparametric Second-Order Efficiency. Ann. Statist. 34, 169-201.

[11] Donoho, D. And Johnstone, I. (1995). Adapting to unknown smoothness via wavelet shrinkage. J. Amer. Statist. Assoc. 90 (432), 1200-1224.

[12] Efromovich, S. Yu. (1999). Nonparametric curve estimation. Methods, theory, and applications. Springer Series in Statistics. Springer-Verlag, New York. 
[13] Fan, J. And Huang, T. (2005). Profile likelihood inferences on semiparametric varying-coefficient partially linear models. Bernoulli 11, 1031-1057.

[14] Gamboa, F., Loubes, J.-M. And MazA, E. (2005). Shifts estimation for high dimensional data. Manuscript downloadable from http://mahery.math.u-psud.fr/ loubes/

[15] Golubev, G. (1990). On estimation of time delay of a signal under nuisance parameters. Probl. Inf. Transm., 25, 3-12.

[16] Golubev, G. And Härdle W. (2000). On the second order minimax estimation in partial linear models. Math. Methods Statist. 2, 160-175.

[17] Golubev, G. And Härdle, W. (2002) On adaptive smoothing in partial linear models. Math. Methods Statist. 1, 98-117.

[18] Härdle, W. AND Marron, J. (1990). Semiparametric comparison of regression curves. Ann. Statist. 18, 63-89.

[19] Härdle, W. And Tsybakov, A. (1993). How sensitive are average derivatives? J. Econometrics, 58, 31-48.

[20] James, W. And Stein, C. (1961). Estimation with quadratic loss. Proc. Forth Berkeley Symp. Math. Statist. Prob. 1, 361-379. Univ. of California Press.

[21] Kang, K., Kim, W. And Park, B. (2000). Higher order kernels in adaptive location estimation. J. Nonparametr. Statist. 12 (6), 879-888.

[22] Lavielle, M. ANd LÉvy-Leduc, C. (2005). Semiparametric estimation of the frequency of unknown periodic functions and its application to laser vibrometry signals. IEEE Transactions on Signal Processing, 53 (7), 2306-2315.

[23] Mammen, E. AND PARK, B. (1997). Optimal smoothing in adaptive location estimation. J. Statist. Plann. Inference 58 (2), 333-348.

[24] Murphy, S. and van der Vaart, A. (2000), On Profile Likelihood. J. Amer. Statist. Assoc. 95, 449-485.

[25] Petrov, V. (1995). Limit theorems of probability theory. Sequences of independent random variables. Oxford Studies in Probability, 4. Oxford University Press, New York.

[26] Pfanzagl, J. (1990). Estimation in Semiparametric Models, Lecture Notes in Statist, 63, Springer, New York.

[27] Pinsker, M. (1980). Optimal filtering of square integrable signals in Gaussian white noise. Probl. Inf. Transm., 16, 120-133.

[28] Rigollet, Ph. (2006). Adaptive density estimation using the blockwise Stein method. Bernoulli, 12 (2), 351-370.

[29] Stein, C. (1956). Inadmissibilty of the usual estimator for the mean of a multivariate normal distribution. Proc. Third Berkeley Symp. Math. Statist. Prob. 1, 197-206. Univ. of California Press.

[30] Schick, A. (1998). Estimating a shift in nonparametric regression via $U$-statistics. J. Statist. Plann. Inference 67 (2), 259-271.

[31] SCHICK, A. (1999). Efficient estimation of a shift in nonparametric regression. Statist. Probab. Lett. 41 (3), 287-301.

[32] Severini, T. And Wong, W. (1992). Profile likelihood and conditionally parametric models. Ann. Statist. 20, 1768-1802.

[33] Stone, C. (1975). Adaptive maximum likelihood estimation of a location parameter. Ann. Statist. 3, 267-284.

[34] Tsybakov, A. (2004). Introduction à l'estimation non-paramétrique. Mathématiques \& Applications, 41. Springer-Verlag, Berlin.

[35] VAN DER VAART, A. (2001). Semiparametric Statistics, manuscript downloadable from http://www.math.vu.nl/sto/publications.php

Université Paris 6, Laboratoire de Probabilités, Boîte courrier 188, 75252 Paris Cedex 05, France, Dalalyan@CCR. Jussieu.fr 